Article

\title{
Optimal Feedback Control Problem for the Fractional Voigt- $\alpha$ Model
}

\author{
Victor Zvyagin $1, *,+\oplus$, Andrey Zvyagin ${ }^{2,+}$ and Anastasiia Ustiuzhaninova ${ }^{3,+}$ \\ 1 Department of Algebra and Mathematical Methods of Fluid Dynamics, Voronezh State University, \\ Universitetskaya pl. 1, 394018 Voronezh, Russia \\ 2 Department of Higher Mathematics, Voronezh State Pedagogical University, Lenina st. 86, \\ 394043 Voronezh, Russia; zvyagin.a@mail.ru \\ 3 Research Institute of Mathematics, Voronezh State University, Universitetskaya pl. 1, \\ 394018 Voronezh, Russia; nastyzhka@gmail.com \\ * Correspondence: zvg_vsu@mail.ru \\ + These authors contributed equally to this work.
}

Received: 14 June 2020; Accepted: 20 July 2020; Published: 21 July 2020

\begin{abstract}
The study of the existence of an optimal feedback control problem for the initial-boundary value problem that describes the motion of the fractional Voigt- $\alpha$ model of a viscoelastic medium is investigated in this paper. In this model, the Voigt rheological relation is considered with the left-side fractional Riemann-Liouville derivative, which allows to take into account the memory of the medium. Also in this model, the memory is considered along the trajectory of the motion of fluid particles, determined by the velocity field. Due to the insufficient smoothness of the velocity field and, as a consequence, the impossibility of uniquely determining the trajectory for the velocity field for any initial value, a weak solution to the problem under study is introduced using regular Lagrangian flows. Based on the approximation-topological approach to the study of fluid dynamic problems, the existence of an optimal solution that gives a minimum to a given cost functional is proved.
\end{abstract}

Keywords: optimal feedback control; Voigt model; alpha-model; fractional derivative

MSC: 76D55; 49J20; 35Q35

\section{Introduction}

The aim of this work is to study the optimal feedback control problem for the alpha-model with the Voigt fractional rheological relation, taking into account the background of a fluid along the trajectory. Note that memory properties in general arise not only in the fluid dynamics field but in many absolute different fields [1]. So the results of this paper can be useful in many fields. A large number of papers have been devoted to the investigation of control problems [2-4]. Although the control problems for linear systems are sufficiently well studied, the situation is not so good for nonlinear systems (even for finite-dimensional cases or local domains). However, due to the complexity of nonlinear systems describing the fluids motion the control of non-Newtonian fluids motion, such as bitumen, polymers, various solutions, emulsions and suspensions, blood, and many others, has not been fully studied. In hydrodynamics the control (optimal control) problems often connected with the fluid control by external forces. Usually in solving such problems, a control is considered from a given (finite) set. In our situation, we consider the external forces control depending on the velocity field. Such types of problems are called feedback control problems [2-5]. In this situation the control is chosen more accurately, since in such a way the control belongs to the image of some multi-valued map. This is more naturally due to the fact that control is not chosen from a finite set of available options. 
Also in this paper the alpha model case of fractional Voigt model is considered. Alpha-models are some kind of regularized approximate systems that depend on some positive parameter $\alpha$, and regularization is carried out by some filtering of the velocity vector, which is contained in the argument of the nonlinear term. The $\alpha$ parameter reflects the width of the spatial filtering scale for the modified speed. The Helmholtz operator $I-\alpha^{2} \Delta$ is most often used as the filtration kernel. The choice of such an operator is associated with its good mathematical properties. Thus, we ready to proceed to the formulation of the problem. In a bounded domain $\Omega \subset \mathbb{R}^{n}$ (in 2D and 3D cases, that is, $n=2,3$ ) with a sufficiently smooth boundary $\partial \Omega$ on a time interval $[0, T]$, where $T>0$, we consider the initial-boundary value problem:

$$
\begin{gathered}
\frac{\partial v}{\partial t}+\sum_{i=1}^{n} u_{i} \frac{\partial v}{\partial x_{i}}-\mu_{0} \Delta v-\frac{\mu_{1}}{\Gamma(1-\beta)} \operatorname{Div} \int_{0}^{t}(t-s)^{-\beta} \mathcal{E}(v)(s, z(s ; t, x)) d s+\nabla p=f, \\
u=\left(I-\alpha^{2} \Delta\right)^{-1} v, \quad t \in[0, T], \quad x \in \Omega, \\
z(\tau ; t, x)=x+\int_{t}^{\tau} v(s, z(s ; t, x)) d s, \quad t, \tau \in[0, T], \quad x \in \Omega, \\
\operatorname{div} v(t, x)=0, \quad t \in[0, T], \quad x \in \Omega, \\
\left.v\right|_{t=0}=v_{0},\left.\quad v\right|_{[0, T] \times \partial \Omega}=0 .
\end{gathered}
$$

Here $v$ is a vector-function of the velocity of a medium particle, $u$ is a vector-function of a modified velocity of a medium particle, defined by equality $(2), z(\tau ; t, x)$ is the trajectory of a medium particle, indicating at time $\tau$ the location of a medium particle located at time moment $t$ at point $x, p$ is a pressure function, $f$ is a function of the density of external forces, $\alpha>0$ is scalar parameter, $\mu_{0}>0$, $\mu_{1} \geq 0,0<\beta<1$ are some constants.

$$
\mathcal{E}=\left(\mathcal{E}_{i j}(v)\right), \quad \mathcal{E}_{i j}(v)=\frac{1}{2}\left(\frac{\partial v_{i}}{\partial x_{j}}+\frac{\partial v_{j}}{\partial x_{i}}\right), \quad i, j=\overline{1, n},
$$

is the strain rate tensor. $\Gamma(\beta)$ is the Euler gamma function [6] defined through an absolutely convergent integral

$$
\Gamma(\beta)=\int_{0}^{\infty} t^{\beta-1} e^{-t} d t
$$

This initial-boundary value problem (1)-(5) is an alpha model for the mathematical model of viscoelastic Voigt medium with fractional rheological relation. The idea of using this kind of approximation (the alpha-model) first appeared in paper of J. Leray [7] (in this work, J. Leray used the general form of the filtration kernel) to prove the existence of a weak solution for the Navier-Stokes system of equations. Later, various alpha-models for the Euler equations [8,9], the Navier-Stokes system [10] and others were built on this idea. In general, each alpha model is characterized by its first-order vector differential operator $F(u, v)=\left(F^{1}(u, v), \ldots, F^{n}(u, v)\right)$, in which components $F^{i}(u, v)$ are linear combinations of all kinds of operators of form $u^{k} \partial_{x_{j}} v^{m}, v^{k} \partial_{x_{j}} u^{m}, u^{k} \partial_{x_{j}} u^{m}$ :

$$
F^{i}(u, v)=\sum_{k, j, m=1}^{n} C_{k j m}^{i} u^{k} \partial_{x_{j}} v^{m}+D_{k j m}^{i} v^{k} \partial_{x_{j}} u^{m}+E_{k j m}^{i} u^{k} \partial_{x_{j}} u^{m},
$$

where $C_{k j m}^{i}, D_{k j m}^{i}, E_{k j m}^{i}$ are some real coefficients. Note that in representation (6) monomials of the form $v^{k} \partial_{x_{j}} v^{m}$ are not used, since they do not contain the components of the «smoothed» vector field $u$.

Interest in the study of alpha-models is primarily associated with their application to the study of turbulence effects for fluid flows. It is also associated with obtaining better numerical results for alpha-models in comparison to the original models. However, most of the works on the solvability of alpha-models are devoted to models of the motion of an ideal or Newtonian fluid [11-14]. Only in the last few years, works began to appear on the study of alpha-models of non-Newtonian fluid [15-18]. 
This work continues the study of alpha-models for non-Newtonian fluids, namely, for the fractional Voigt model of the viscoelastic medium [19]. This mathematical model describes a viscoelastic fluid flow with a rheological relation $\sigma=\mu_{0} \mathcal{E}(v)+\mu_{1} D_{0 t}^{\beta} \mathcal{E}(v)=\mu_{0} \mathcal{E}(v)+\mu_{1} I_{0 t}^{1-\beta} \mathcal{E}(v)$, considered along the trajectories of fluid motion. Here $D_{0 t}^{\beta}$ is the left-side fractional Riemann-Liouville derivative and $I_{0 t}^{1-\beta}$ is the Riemann-Liouville fractional integral. This model is a fractional analog of the Voigt model, which describes the motion of a linearly elastic-retarded fluid. In order to study a large class of polymers with creep and relaxation effects one must to consider models with fractional derivatives. It turns out that the models with fractional derivatives are most suitable for this [20,21]. Note that the advantage of this model is that, together with the definition of the vector-velocity $v$ of the particle's motion, the trajectory of the particles of this medium motion $z$ is also determined. Also, note that the consideration of fractional derivatives in fluid dynamics has many physical applications [22-24]. One of the possible continuations of this model studies is laid out in References [25] and [26].

\section{Preliminary Information and Statement of the Main Results}

We introduce the main notation and auxiliary statements.

By $L_{p}(\Omega), 1 \leq p<\infty$, we denote the set of measurable vector functions $v: \Omega \rightarrow \mathbb{R}^{n}$, summable with $p$ degree. By $W_{p}^{m}(\Omega), m \geq 1, p \geq 1$, we denote Sobolev spaces. We consider the space $C_{0}^{\infty}(\Omega)^{n}$ of infinitely differentiable vector functions from $\Omega$ to $\mathbb{R}^{n}$ with compact support in $\Omega$. Denote by $\mathcal{V}$ the set $\left\{v \in C_{0}^{\infty}(\Omega)^{n}, \operatorname{div} v=0\right\}$. Also by $V^{0}$ and $V^{1}$ we denote the closure of $\mathcal{V}$ with respect to the norm of $L_{2}(\Omega)$ and $W_{2}^{1}(\Omega)$, respectively, and by $V^{2}$ we denote the space $V^{2}=W_{2}^{2}(\Omega) \cap V^{1}$.

We introduce from Reference [27] the scale of spaces $V^{\beta}, \beta \in \mathbb{R}$. For this we consider the Leray projector $P: L_{2}(\Omega) \rightarrow V^{0}$ and the operator $A=-P \Delta$ defined on $D(A)=V^{2}$. From this operator we can get a self-adjoint positive operator with compact inverse in $V^{0}$. Let $0<\lambda_{1} \leq \lambda_{2} \leq \cdots \leq \lambda_{k} \leq \ldots$ be the eigenvalues of the operator $A$. We can get an orthonormal basis in $V^{0}$ by the eigenfunctions $\left\{e_{j}\right\}$ of the operator $A$ due to the Hilbert theorem on the spectral decomposition of compact operators. Denote by

$$
E_{\infty}=\left\{v=\sum_{j=1}^{N} v_{j} e_{j}: v_{j} \in \mathbb{R}, N \in \mathbb{N}\right\}
$$

the set of finite linear combinations of $e_{j}$. Thus, we get the space $V^{\beta}, \beta \in \mathbb{R}$ as the completion of $E_{\infty}$ with respect to the norm

$$
\|v\|_{V^{\beta}}=\left(\sum_{k=1}^{\infty} \lambda_{k}^{\beta}\left|v_{k}\right|^{2}\right)^{\frac{1}{2}} .
$$

In Reference [27] it is shown that on the space $V^{\beta}, \beta>-1 / 2$, norm (7) is equivalent to the ordinary norm $\|\cdot\|_{W_{2}^{\beta}(\Omega)^{n}}$ of the space $W_{2}^{\beta}(\Omega)^{n}$. In addition, according to Reference [28], the norms in the spaces $V^{1}, V^{2}$ and $V^{3}$ can be defined as follows:

$$
\begin{gathered}
\|v\|_{V^{1}}=\left(\int_{\Omega} \nabla v(x): \nabla v(x) \mathrm{d} x\right)^{\frac{1}{2}}, \quad\|v\|_{V^{2}}=\left(\int_{\Omega} \Delta v(x) \Delta v(x) \mathrm{d} x\right)^{\frac{1}{2}}, \\
\|v\|_{V^{3}}=\left(\int_{\Omega} \nabla \Delta v(x): \nabla \Delta v(x) \mathrm{d} x\right)^{\frac{1}{2}} .
\end{gathered}
$$

Here the symbol " : " denotes the component-wise matrix product, that is, for $C=\left(c_{i j}\right), D=\left(d_{i j}\right)$, $i, j=1, \ldots m$, we put $C: D=\sum_{i, j=1}^{m} c_{i j} d_{i j}$.

Further, through the $V^{-\beta}=\left(V^{\beta}\right)^{*}, \beta \in \mathbb{N}$, we denote the space dual to $V^{\beta}$. 
Note that $C([0, T] ; F)$ is the Banach space of continuous on $[0, T]$ functions, $C_{w}([0, T] ; F)$ is the Banach space of weakly continuous on $[0, T]$ functions, $L_{p}(0, T ; F)$ is the Banach spaces of summable on $[0, T]$ with $p$ degree functions with values in a Banach space $F$, respectively.

The set $C^{1} D(\bar{\Omega})$ consists of one-to-one mappings $z: \bar{\Omega} \rightarrow \bar{\Omega}$ coinciding with the identity mapping on $\partial \Omega$ and having continuous first-order partial derivatives on $\Omega$ such that $\operatorname{det}(\partial z / \partial x)=1$ at every point of the domain $\Omega$. For this set the norm of continuous functions $C(\bar{\Omega})$ is used. Further, we will consider the following set $C G=C\left([0, T] \times[0, T], C^{1} D(\bar{\Omega})\right)$. Note that $C G \subset C\left([0, T] \times[0, T], C^{1}(\bar{\Omega})\right)$, therefore, in what follows, $C G$ is considered a metric space with a metric defined by the norm of the space $C([0, T] \times[0, T], C(\bar{\Omega}))$.

We introduce the space in which the solvability of the considered problem will be proved:

$$
W_{1}=\left\{v \in L_{2}\left(0, T ; V^{1}\right) \cap L_{\infty}\left(0, T ; V^{0}\right), \quad v^{\prime} \in L_{4 / 3}\left(0, T ; V^{-1}\right)\right\}
$$

with the norm $\|v\|_{W_{1}}=\|v\|_{L_{2}\left(0, T ; V^{1}\right)}+\|v\|_{L_{\infty}\left(0, T ; V^{0}\right)}+\left\|v^{\prime}\right\|_{L_{4 / 3}\left(0, T ; V^{-1}\right)}$.

Denote by $\Delta_{\alpha}: V^{\beta} \rightarrow V^{\beta-2}, \beta \geq 0$ the operator $\Delta_{\alpha}=\left(J+\alpha^{2} A\right)$, where $J=P I$, and $I$ is the identity operator. By virtue of Reference [28], the operator $\Delta_{\alpha}$ is invertible. If we apply the Leray projection $P: L_{2}(\Omega) \rightarrow V^{0}$ to the equality $v=\left(I-\alpha^{2} \Delta\right) u$ for $\beta=3$ and express from the last equality $u: u=\left(J+\alpha^{2} A\right)^{-1} v=\Delta_{\alpha}^{-1} v$. Then, since $v \in V^{1}$, we get that $u \in V^{3}$.

Note that for the correct formulation of the considered initial-boundary value problem the trajectories $z$ must be uniquely determined by the velocity field $v$. In other words, it is necessary that Equation (3) has a unique solution for the velocity field $v$. However, the existence of solutions to Equation (3) for a fixed $v$ is known in Reference [29] only in case $v \in L_{1}\left(0, T ; C(\bar{\Omega})^{n}\right)$ and this solution is unique for $v \in L_{1}\left(0, T ; C^{1}(\bar{\Omega})^{n}\right)$ such that $\left.v\right|_{(0, T) \times \partial \Omega}=0$. Therefore, the trajectories of motion are not uniquely determined even for strong solutions whose partial derivatives that appear in Equation (3) are contained in $L_{2}\left(0, T ; L_{2}(\Omega)\right)$. One possible way out of this situation is to regularize the velocity field at each time instant $t$ by averaging over the variable $x$ and determine the trajectories $z(\tau ; t, x)$ for the regularized velocity field [30]. However, relatively recently [31,32], the solvability of Cauchy integral problem (3) was investigated in the case when the velocity $v$ belongs to the Sobolev space. Also the existence and uniqueness of regular Lagrangian flows, which are a generalization of the concept of a classical solution, are established.

Definition 1. Regular Lagrangian flow associated to $v$ is the function $z(\tau ; t, x),(\tau ; t, x) \in[0, T] \times[0, T] \times \bar{\Omega}$ satisfying conditions:

1. the function $\gamma(\tau)=z(\tau ; t, x)$ is absolutely continuous and satisfies Equation (3) for almost all $x \in \bar{\Omega}$ and $t \in[0, T] ;$

2. the equality $m(z(\tau ; t, B))=m(B)$ holds for any $t, \tau \in[0, T]$ and an arbitrary Lebesgue measurable set $B \subseteq \bar{\Omega}$ with Lebesgue measure $m(B)$;

3. for all $t_{i} \in[0, T], i=1,2,3$, and almost all $x \in \bar{\Omega}$

$$
z\left(t_{3} ; t_{1}, x\right)=z\left(t_{3} ; t_{2}, z\left(t_{2} ; t_{1}, x\right)\right)
$$

We give the necessary results from a regular Lagrangian flow.

Theorem 1. [31] Let $v \in L_{1}\left(0, T ; W_{p}^{1}(\Omega)^{n}\right), 1 \leq p \leq \infty$ with conditions $\operatorname{div} v(t, x)=0,(t, x) \in[0, T] \times \Omega$, and $\left.v\right|_{[0, T] \times \partial \Omega}=0$. Then there exists a unique regular Lagrangian flow $z \in C\left(D ; L^{n}\right)$ associated to $v$ (where $C(D, L)$ is the Banach space of continuous functions on $D=[0, T] \times[0, T]$ with values in the metric space of vector functions $L$ measurable on $\Omega$ ). Moreover, $z(\tau ; t, \bar{\Omega}) \subset \bar{\Omega}$ up to a set of measure zero and

$$
\frac{\partial}{\partial \tau} z(\tau ; t, x)=v(\tau, z(\tau ; t, x)), \quad t, \tau \in[0, T], \text { for almost all } x \in \Omega .
$$


Theorem 2. Let $v, v^{m} \in L_{1}\left(0, T ; W_{1}^{p}(\Omega)^{n}\right), m=1,2, \ldots$ for some $p>1$. Let $\operatorname{div} v=0$, div $v^{m}=0$, $\left.v\right|_{[0, T] \times \partial \Omega}=0,\left.v^{m}\right|_{[0, T] \times \partial \Omega}=0$. Also, let the inequalities

$$
\begin{gathered}
\left\|v_{x}\right\|_{L_{1}\left(0, T ; L_{p}(\Omega)^{n \times n}\right)}+\|v\|_{L_{1}\left(0, T ; L_{p}(\Omega)^{n}\right)} \leq M \\
\left\|v_{x}^{m}\right\|_{L_{1}\left(0, T ; L_{p}(\Omega)^{n \times n}\right)}+\left\|v^{m}\right\|_{L_{1}\left(0, T ; L_{p}(\Omega)^{n}\right)} \leq M
\end{gathered}
$$

are valid. Here $v_{x}$ and $v_{x}^{m}$ are the Jacobi matrices of the vector functions $v$ and $v^{m}$. Let $v^{m}$ converges to $v$ in $L_{1}\left(Q_{T}\right)^{N}$ as $m \rightarrow+\infty$. Let $z^{m}(\tau ; t, x)$ and $z(\tau ; t, x)$ are regular Lagrangian flows associated to $v^{m}$ and $v$, respectively. Then the sequence $z^{m}$ converges (up to a subsequence) to $z$ with respect to the Lebesgue measure on the set $[0, T] \times \Omega$ uniformly on $t \in[0, T]$.

This result was proved in Reference [33] in the general case.

Thus, by virtue of Theorem 1 for each $v \in L_{2}\left(0, T ; V^{1}\right)$ and for almost all $x \in \Omega$, the Equation (3) has a unique solution $z(v)$, where $z(v)(\tau ; t, x)=z(\tau ; t, x)$, in the class of regular Lagrangian flows.

As a control function, we consider the multi-valued map $\Psi: W_{1} \multimap L_{2}\left(0, T, V^{-1}\right)$. Assume that $\Psi$ satisfies the following conditions:

( $\Psi 1) \quad \Psi$ is defined on the space $W_{1}$ and has nonempty, compact, and convex values;

( $\Psi 2) \quad \Psi$ is compact and upper semicontinuous (that is, for any function $v \in W_{1}$ and any open set $Y \subset L_{2}\left(0, T, V^{-1}\right)$ such that $\Psi(v) \subset Y$, there exists a neighborhood $U(v)$ such that $\Psi(U(v)) \subset Y)$;

(ษ3) $\Psi$ is globally bounded, that is, there exists a constant $R_{1}>0$ such that

$$
\|\Psi(v)\|_{L_{2}\left(0, T, V^{-1}\right)}:=\sup \left\{\|u\|_{L_{2}\left(0, T, V^{-1}\right)}: u \in \Psi(v)\right\} \leq R_{1} \text { for all } v \in W_{1} ;
$$

( $\Psi 4) \quad \Psi$ is weakly closed, that is: if $\left\{v_{l}\right\}_{l=1}^{\infty} \subset W_{1}, v_{l} \rightarrow v_{0}, u_{l} \in \Psi\left(v_{l}\right)$ and $u_{l} \rightarrow u_{0}$ in $L_{2}\left(0, T, V^{-1}\right)$ then $u_{0} \in \Psi\left(v_{0}\right)$.

In this paper, a weak statement of the feedback control problem for initial-boundary value problem (1)-(5) is considered. By feedback, we mean the condition

$$
f \in \Psi(v)
$$

We formulate the definition of a weak solution to feedback control problem (1)-(5), (8):

Definition 2. A pair of functions $(v, f) \in W_{1} \times L_{2}\left(0, T, V^{-1}\right)$ is called a weak solution of feedback control problem (1)-(5), (8), if it for all $\varphi \in V^{1}$ and almost all $t \in(0, T)$ satisfies the equality

$$
\begin{aligned}
\left\langle v^{\prime}, \varphi\right\rangle-\int_{\Omega} \sum_{i, j=1}^{n}\left(\Delta_{\alpha}^{-1} v\right)_{i} v_{j} \frac{\partial \varphi_{j}}{\partial x_{i}} & d x+\mu_{0} \int_{\Omega} \nabla v: \nabla \varphi d x \\
& +\frac{\mu_{1}}{\Gamma(1-\beta)}\left(\int_{0}^{t}(t-s)^{-\beta} \mathcal{E}(v)(s, z(v)(s ; t, x)) d s, \mathcal{E}(\varphi)\right)=\langle f, \varphi\rangle,
\end{aligned}
$$

the initial condition $v(0)=v_{0}$ and feedback condition (8). Here $z(v)$ is a regular Lagrangian flow associated to $v$.

Remark 1. It is known that $W \subset C_{w}\left(0, T ; V^{0}\right)$ [34]. Therefore, initial condition (5) has sense.

The following theorem is the first result of the paper:

Theorem 3. Let a multi-valued mapping $\Psi$ satisfy conditions $(\Psi 1)-(\Psi 4)$. Then there is at least one weak solution $\left(v_{*}, f_{*}\right) \in W_{1} \times L_{2}\left(0, T, V^{-1}\right)$ of feedback control problem (1)-(5), (8). 
We denote by $\Sigma \subset W_{1} \times L_{2}\left(0, T ; V^{-1}\right)$ the set of all weak solutions of problem (1)-(5), (8). Consider an arbitrary cost functional $\Phi: \Sigma \rightarrow \mathbb{R}$, satisfying the following conditions:

(Ф1) For all $(v, f) \in \Sigma$ a number $R_{2}$ exists such that $\Phi(v, f) \geq R_{2}$.

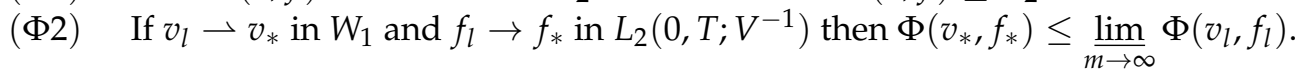

As an example of this functional, we can take

$$
\Phi(v, f)=\int_{0}^{T}\left\|v(t)-u_{*}(t)\right\|_{V^{1}}^{2} d t+\int_{0}^{T}\|f(t)\|_{V^{-1}}^{2} d t
$$

Here $u_{*}$ is some specified velocity field. This functional characterizes the deviation of velocity from the required, and its minimum yields the minimal deviation of velocity from the one specified by the minimal control. One of the possible applications of the proposed approach is an optimal feedback control problem and the results are in the consideration, analysis and calculation of different such problems with special (necessary in industry) cost functionals $\Phi$.

The following theorem is the second result of this paper.

Theorem 4. If the mapping $\Psi$ satisfies conditions $(\Psi 1)-(\Psi 4)$ and the functional $\Phi$ satisfies conditions $(\Phi 1),(\Phi 2)$, then optimal feedback control problem $(1)-(5),(8)$ has at least one weak solution $\left(v_{*}, f_{*}\right)$ such that

$$
\Phi\left(v_{*}, f_{*}\right)=\inf _{(v, f) \in \Sigma} \Phi(v, f) .
$$

The proof of Theorems 3 and 4 is based on the approximation-topological method for investigating fluid dynamics problems [35]. To do this, first, we pass to the operator interpretation of the problem under consideration (operator inclusion) in suitable function spaces. Further, since the operators in the obtained operator inclusion do not have the necessary properties, we consider a problem that approximates the original one (in this case, it is also an operator inclusion, but with a better operator that has the required properties and in better functional spaces). Then, based on a priori estimates of solutions and the theory of the topological degree of multi-valued vector fields, the existence of a solution to the approximation problem is proved. Finally, it is shown that from the sequence of solutions of the approximation problem, one can extract a subsequence that converges in a weak sense to the solution of the original operator inclusion. After proving the solvability of the control problem, it is shown that in the set of solutions there is at least one solution that gives a minimum to a given cost functional (this is why this type of problem is called the optimal feedback control problem for fluid motion).

The work is organized as follows-in Section 3 we consider the family of auxiliary problems and prove the necessary properties of an introduced operators. Also on the basis of the topological degree theory for multivalued maps we prove the solvability of the auxiliary problem and establish necessary estimates for solutions to the auxiliary problem. Section 4 is devoted to the passage, the limit and the proof of Theorem 3. Section 5 is devoted to the proof of Theorem 4 . The final Section 6 contains conclusions.

\section{The Family of Auxiliary Problems}

Throughout this section we will assume that $v_{0} \in V^{3}$. 
Consider the following auxiliary family of systems of equations $(0 \leq \xi \leq 1)$ with a small parameter $\varepsilon>0$ :

$$
\begin{gathered}
\varepsilon \frac{\partial \Delta^{2} v}{\partial t}+\frac{\partial v}{\partial t}+\xi \sum_{i=1}^{n}\left(\Delta_{\alpha}^{-1} v\right)_{i} \frac{\partial v}{\partial x_{i}}-\mu_{0} \Delta v \\
-\frac{\mu_{1} \xi}{\Gamma(1-\beta)} \operatorname{Div} \int_{0}^{t}(t-s)^{-\beta} \mathcal{E}(v)(s, z(s ; t, x)) d s+\nabla p=\xi f, \\
z(\tau ; t, x)=x+\int_{t}^{\tau} v(s, z(s ; t, x)) d s, \quad t, \tau \in[0, T], \quad x \in \Omega, \\
\operatorname{div} v=0, \quad t \in[0, T], \quad x \in \Omega, \\
\left.v\right|_{\partial \Omega}=0,\left.\quad \Delta v\right|_{\partial \Omega}=0, \quad t \in[0, T] \\
\left.v\right|_{t=0}=v_{0}, \quad x \in \Omega .
\end{gathered}
$$

For this family we consider another functional space:

$$
W_{2}=\left\{v \in C\left([0, T] ; V^{3}\right), \quad v^{\prime} \in L_{2}\left(0, T ; V^{3}\right)\right\}
$$

with the norm $\|v\|_{W_{2}}=\|v\|_{C\left(0, T ; V^{3}\right)}+\left\|v^{\prime}\right\|_{L_{2}\left(0, T ; V^{3}\right)}$.

Equation (10) includes the integral calculated along the trajectories of motion of the fluid particles. As was noted in the previous section, it is necessary that the trajectories are uniquely determined by the velocity field $v(t, x)$. In other words, Equation (11) must have a unique solution for the velocity field $v(t, x)$. Note that for the family of auxiliary problems (10)-(14), the velocity $v$ from the space $W_{2}$ has sufficient smoothness (due to the embedding of the space $V^{3}$ in $C^{1}(\bar{\Omega})$ for $n=2,3$ ). Thus, it follows from Reference [29] that the Cauchy problem (11) is non-locally uniquely solvable.

Analogously with the definition of a weak solution for feedback control problem (1)-(5), (8), we formulate the definition of a weak solution to auxiliary problem (10)-(14), (8) for fixed $0 \leq \xi \leq 1$.

Definition 3. A pair of functions $(v, f) \in W_{2} \times L_{2}\left(0, T ; V^{-1}\right)$ is called a weak solution to auxiliary problem (10)-(14), (8) if it satisfies for any $\varphi \in V^{1}$ and almost all $t \in(0, T)$ the equality

$$
\begin{aligned}
\left\langle v^{\prime}, \varphi\right\rangle & -\xi \int_{\Omega} \sum_{i, j=1}^{n}\left(\Delta_{\alpha}^{-1} v\right)_{i} v_{j} \frac{\partial \varphi_{j}}{\partial x_{i}} d x+\mu_{0} \int_{\Omega} \nabla v: \nabla \varphi d x-\varepsilon \int_{\Omega} \nabla \Delta v^{\prime}: \nabla \varphi d x \\
& +\frac{\mu_{1} \xi}{\Gamma(1-\beta)}\left(\int_{0}^{t}(t-s)^{-\beta} \mathcal{E}(v)(s, z(s ; t, x)) d s, \mathcal{E}(\varphi)\right)=\xi\langle f, \varphi\rangle,
\end{aligned}
$$

feedback condition (8) and initial condition (14). Here $z$ is the trajectory associated to the velocity $v$. 
To prove the existence of a weak solution to auxiliary problem (10)-(14),(8) for $\xi=1$, we rewrite the auxiliary family in operator form. Using the terms in equality (15), we introduce the operators using the following equalities:

$$
\begin{gathered}
J: V^{3} \rightarrow V^{-1}, \quad\langle J v, \varphi\rangle=\int_{\Omega} v \varphi d x, \quad v \in V^{3}, \quad \varphi \in V^{1} ; \\
A: V^{1} \rightarrow V^{-1}, \quad\langle A v, \varphi\rangle=\int_{\Omega} \nabla v: \nabla \varphi d x, \quad v \in V^{1}, \quad \varphi \in V^{1} ; \\
A_{2}: V^{3} \rightarrow V^{-1}, \quad\left\langle A_{2} v, \varphi\right\rangle=-\int_{\Omega} \nabla \Delta v: \nabla \varphi d x, \quad v \in V^{3}, \quad \varphi \in V^{1} ; \\
B: L_{4}(\Omega) \rightarrow V^{-1}, \quad\langle B(v), \varphi\rangle=\int_{\Omega} \sum_{i, j=1}^{n}\left(\Delta_{\alpha}^{-1} v\right)_{i} v_{j} \frac{\partial \varphi_{j}}{\partial x_{i}} d x, \quad v \in L_{4}(\Omega), \quad \varphi \in V^{1} ; \\
C: V^{1} \times C G \rightarrow V^{-1}, \quad(C(v, z)(t), \varphi)=\left(\int_{0}^{t}(t-s)^{-\beta} \mathcal{E}(v)(s, z(s ; t, x)) d s, \mathcal{E}(\varphi)\right), \\
v \in V^{1}, z \in C G, \varphi \in V^{1}, \text { for almost all } t \in(0, T) .
\end{gathered}
$$

Since the function $\varphi \in V^{1}$ is arbitrary in (15), for almost all $t \in(0, T)$ this equality is equivalent to the following operator equation in $L_{2}\left(0, T ; V^{-1}\right)$ :

$$
J v^{\prime}+\varepsilon A_{2} v^{\prime}+\mu_{0} A v-\xi B(v)+\frac{\mu_{1} \xi}{\Gamma(1-\beta)} C(v, z)=\xi f .
$$

Thus, a weak solution to auxiliary problem (10)-(14), (8) for a fixed $0 \leq \xi \leq 1$ is a solution $v \in W_{2}$ of the following operator inclusion

$$
J v^{\prime}+\varepsilon A_{2} v^{\prime}+\mu_{0} A v-\xi B(v)+\frac{\mu_{1} \xi}{\Gamma(1-\beta)} C(v, z)=\xi f \in \Psi(v),
$$

satisfying initial condition (14).

We also define the operators using the following equalities:

$$
\begin{gathered}
L: W_{2} \rightarrow L_{2}\left(0, T ; V^{-1}\right) \times V^{3}, \quad L(v)=\left(\left(J+\varepsilon A_{2}\right) v^{\prime}+\mu_{0} A v,\left.v\right|_{t=0}\right) \\
K: W_{2} \rightarrow L_{2}\left(0, T ; V^{-1}\right) \times V^{3}, \quad K(v)=(B(v), 0) ; \\
G: W_{2} \rightarrow L_{2}\left(0, T ; V^{-1}\right) \times V^{3}, \quad G(v)=\left(\frac{\mu_{1}}{\Gamma(1-\beta)} C(v, z), 0\right) ; \\
\mathcal{Y}: W_{2} \rightarrow L_{2}\left(0, T ; V^{-1}\right) \times V^{3}, \quad \mathcal{Y}(v)=\left(\Psi(v), v_{0}\right) ; \\
\mathcal{M}: W_{2} \rightarrow W_{2}, \quad \mathcal{M}(v)=L^{-1}(\mathcal{Y}(v)+K(v)-G(v)) .
\end{gathered}
$$

Thus, from our problem of finding a solution to operator inclusion (16) for a fixed $0 \leq \xi \leq 1$ satisfying initial condition (14) we get the problem of finding a solution for a fixed $0 \leq \xi \leq 1$ to the following operator inclusion

$$
v \in \xi \mathcal{M}(v)=\xi L^{-1}(\mathcal{Y}(v)+K(v)-G(v)) .
$$

We need the following properties of the operators from inclusions (16) and (17). In order to not to pile up the notation, we will use the same letter to denote the same operators acting in different function spaces.

Lemma 1. 1. For any function $v \in C\left([0, T] ; V^{3}\right)$ it holds that the function $A v \in L_{2}\left(0, T ; V^{-1}\right)$ and the operator $A: C\left([0, T] ; V^{3}\right) \rightarrow L_{2}\left(0, T ; V^{-1}\right)$ is continuous and the following estimates hold:

$$
\|A v\|_{V^{-1}} \leq\|v\|_{V^{1}} ; \quad\|A v\|_{L_{2}\left(0, T ; V^{-1}\right)} \leq\|v\|_{L_{2}\left(0, T ; V^{1}\right)} ;
$$




$$
\|A v\|_{L_{2}\left(0, T ; V^{-1}\right)} \leq C_{1}\|v\|_{C\left([0, T] ; V^{3}\right)} .
$$

2. The operator $A_{2}: V^{3} \rightarrow V^{-1}$ is linear, continuous, invertible and the following estimate holds:

$$
\left\|A_{2} v\right\|_{V^{-1}} \leq\|v\|_{V^{3}}
$$

In addition, the operator $A_{2}^{-1}: V^{-1} \rightarrow V^{3}$ is also continuous.

3. For any function $v \in L_{p}\left(0, T ; V^{3}\right), 1 \leq p<\infty$ the function $\left(J+\varepsilon A_{2}\right) v$ belongs to $L_{p}\left(0, T ; V^{-1}\right)$ and the operator $\left(J+\varepsilon A_{2}\right): L_{p}\left(0, T ; V^{3}\right) \rightarrow L_{p}\left(0, T ; V^{-1}\right)$ is continuous and invertible. In addition, the following estimate holds:

$$
\varepsilon\|v\|_{L_{p}\left(0, T ; V^{3}\right)} \leq\left\|\left(J+\varepsilon A_{2}\right) v\right\|_{L_{p}\left(0, T ; V^{-1}\right)} \leq C_{2}(1+\varepsilon)\|v\|_{L_{p}\left(0, T ; V^{3}\right)} .
$$

Moreover, the inverse to it operator $\left(J+\varepsilon A_{2}\right)^{-1}: L_{p}\left(0, T ; V^{-1}\right) \rightarrow L_{p}\left(0, T ; V^{3}\right)$ is continuous and for any $w \in L_{p}\left(0, T ; V^{-1}\right)$ we have the estimate

$$
\left\|\left(J+\varepsilon A_{2}\right)^{-1} w\right\|_{L_{p}\left(0, T ; V^{3}\right)} \leq \frac{1}{\varepsilon}\|w\|_{L_{p}\left(0, T ; V^{-1}\right)} .
$$

4. The operator $L: W_{2} \rightarrow L_{2}\left(0, T ; V^{-1}\right) \times V^{3}$ is invertible and the operator $L^{-1}: L_{2}\left(0, T ; V^{-1}\right) \times V^{3} \rightarrow$ $W_{2}$ is a continuous operator.

Proof. The proof is carried out in the same way as in Reference [36].

Lemma 2. 1. The map $B: L_{4}(\Omega) \rightarrow V^{-1}$ is continuous and the following estimate holds:

$$
\|B(v)\|_{V^{-1}} \leq C_{3}\|v\|_{L_{4}(\Omega)}^{2} .
$$

2. For any $v \in L_{4}\left(0, T ; L_{4}(\Omega)\right)$ the function $B(v) \in L_{2}\left(0, T ; V^{-1}\right)$ and the map $B: L_{4}\left(0, T ; L_{4}(\Omega)\right) \rightarrow$ $L_{2}\left(0, T ; V^{-1}\right)$ is continuous.

3. For any function $v \in W_{2}$ the function $B(v) \in L_{2}\left(0, T ; V^{-1}\right)$ and the map $B: W_{2} \rightarrow L_{2}\left(0, T ; V^{-1}\right)$ is compact.

Proof. 1. For any $v \in L_{4}(\Omega), \varphi \in V^{1}$ using Holder's inequality, we obtain

$$
\begin{gathered}
|\langle B(v), \varphi\rangle|=\left|\sum_{i, j=1}^{n} \int_{\Omega}\left(\Delta_{\alpha}^{-1} v\right)_{i} v_{j} \frac{\partial \varphi_{j}}{\partial x_{i}} d x\right| \leq \sum_{i, j=1}^{n}\left(\int_{\Omega}\left|\left(\Delta_{\alpha}^{-1} v\right)_{i} v_{j}\right|^{2} d x\right)^{\frac{1}{2}} \\
\times\left(\int_{\Omega}\left|\frac{\partial \varphi_{j}}{\partial x_{i}}\right|^{2} d x\right)^{\frac{1}{2}} \leq \sum_{i, j=1}^{n}\left(\int_{\Omega}\left|\left(\Delta_{\alpha}^{-1} v\right)_{i}\right|^{4} d x\right)^{\frac{1}{4}}\left(\int_{\Omega}\left|v_{j}\right|^{4} d x\right)^{\frac{1}{4}}\|\varphi\|_{V^{1}} \\
\leq C_{4}\left\|\Delta_{\alpha}^{-1} v\right\|_{L_{4}(\Omega)}\|v\|_{L_{4}(\Omega)}\|\varphi\|_{V^{1}} \leq C_{4} C_{5}\|v\|_{L_{4}(\Omega)}^{2}\|\varphi\|_{V^{1}}=C_{6}\|v\|_{L_{4}(\Omega)}^{2}\|\varphi\|_{V^{1}} .
\end{gathered}
$$

This implies inequality (23). Note that here we used the following well-known estimate $[37,38]$ :

$$
\left\|\Delta_{\alpha}^{-1} v\right\|_{L_{p}(\Omega)}=\left\|\left(I-\alpha^{2} \Delta\right)^{-1} v\right\|_{L_{p}(\Omega)} \leq C_{5}\|v\|_{L_{p}(\Omega)}, \quad p>1 .
$$


We show the continuity of the map $B: L_{4}(\Omega) \rightarrow V^{-1}$. For arbitrary $v^{m}, v^{0} \in L_{4}(\Omega)$ we have

$$
\begin{gathered}
\left|\left\langle B\left(v^{m}\right), \varphi\right\rangle-\left\langle B\left(v^{0}\right), \varphi\right\rangle\right|=\left|\int_{\Omega} \sum_{i, j=1}^{n}\left(\Delta_{\alpha}^{-1} v^{m}\right)_{i} v_{j}^{m} \frac{\partial \varphi_{j}}{\partial x_{i}} d x-\int_{\Omega} \sum_{i, j=1}^{n}\left(\Delta_{\alpha}^{-1} v^{0}\right)_{i} v_{j}^{0} \frac{\partial \varphi_{j}}{\partial x_{i}} d x\right| \\
\leq \sum_{i, j=1}^{n}\left\|\left(\Delta_{\alpha}^{-1} v^{m}\right)_{i} v_{j}^{m}-\left(\Delta_{\alpha}^{-1} v^{0}\right)_{i} v_{j}^{0}\right\|_{L_{2}(\Omega)}\left\|\frac{\partial \varphi_{j}}{\partial x_{i}}\right\|_{L_{2}(\Omega)} \\
\leq\|\varphi\|_{V^{1}} \sum_{i, j=1}^{n}\left\|\left(\Delta_{\alpha}^{-1} v^{m}\right)_{i} v_{j}^{m}-\left(\Delta_{\alpha}^{-1} v^{0}\right)_{i} v_{j}^{0}\right\|_{L_{2}(\Omega)} \\
=\|\varphi\|_{V^{1}}\left(\sum_{i, j=1}^{n}\left\|\left(\Delta_{\alpha}^{-1} v^{m}\right)_{i} v_{j}^{m}-\left(\Delta_{\alpha}^{-1} v^{m}\right)_{i} v_{j}^{0}+\left(\Delta_{\alpha}^{-1} v^{m}\right)_{i} v_{j}^{0}-\left(\Delta_{\alpha}^{-1} v^{0}\right)_{i} v_{j}^{0}\right\|_{L_{2}(\Omega)}\right) \\
\leq\|\varphi\|_{V^{1}}\left(\sum_{i, j=1}^{n}\left\|\left(\Delta_{\alpha}^{-1} v^{m}\right)_{i} v_{j}^{m}-\left(\Delta_{\alpha}^{-1} v^{m}\right)_{i} v_{j}^{0}\right\|_{L_{2}(\Omega)}+\sum_{i, j=1}^{n}\left\|\left(\Delta_{\alpha}^{-1} v^{m}\right)_{i} v_{j}^{0}-\left(\Delta_{\alpha}^{-1} v^{0}\right)_{i} v_{j}^{0}\right\|_{L_{2}(\Omega)}\right) \\
\leq C_{7}\|\varphi\|_{V^{1}}\left(\sum_{j=1}^{n}\left\|\Delta_{\alpha}^{-1} v^{m}\right\|_{L_{4}(\Omega)}\left\|v_{j}^{m}-v_{j}^{0}\right\|_{L_{4}(\Omega)}+\sum_{j=1}^{n}\left\|\Delta_{\alpha}^{-1}\left(v^{m}-v^{0}\right)\right\|_{L_{4}(\Omega)}\left\|_{v_{j}^{0}}\right\|_{L_{4}(\Omega)}\right) \\
\leq C_{7} C_{5}\|\varphi\|_{V^{1}}\left(\sum_{j=1}^{n}\left\|v^{m}\right\|_{L_{4}(\Omega)}\left\|v_{j}^{m}-v_{j}^{0}\right\|_{L_{4}(\Omega)}+\sum_{j=1}^{n}\left\|v^{m}-v^{0}\right\|_{L_{4}(\Omega)}\left\|v_{j}^{0}\right\|_{L_{4}(\Omega)}\right) \\
\leq C_{8}\left(\left\|v^{m}\right\|_{L_{4}(\Omega)}\left\|v^{m}-v^{0}\right\|_{L_{4}(\Omega)}+\left\|v^{m}-v^{0}\right\| L_{L_{4}(\Omega)}\left\|v^{0}\right\|_{L_{4}(\Omega)}\right)\|\varphi\|_{V^{1}} \\
=C_{8}\left(\left\|v^{m}\right\|_{L_{4}(\Omega)}+\left\|v^{0}\right\|_{L_{4}(\Omega)}\right)\left\|v^{m}-v^{0}\right\|_{L_{4}(\Omega)}\|\varphi\|_{V^{1}} .
\end{gathered}
$$

Thereby

$$
\left\|B\left(v^{m}\right)-B\left(v^{0}\right)\right\|_{V^{-1}} \leq C_{8}\left(\left\|v^{m}\right\|_{L_{4}(\Omega)}+\left\|v^{0}\right\|_{L_{4}(\Omega)}\right)\left\|v^{m}-v^{0}\right\|_{L_{4}(\Omega)} .
$$

Assuming that $v^{m} \rightarrow v^{0}$ in $L_{4}(\Omega)$, we obtain that the map $B: L_{4}(\Omega) \rightarrow V^{-1}$ is continuous.

2. To prove this item, it is necessary to use the last estimate and repeat the proof of Lemma 2.5.4 (item 2) from Reference [28].

3. To prove this item, we use the Aubin-Simon theorem:

Theorem 5. $[28,39,40]$ Let $X \subset E \subset Y$ are Banach spaces, the embedding $X \subset E$ is compact and the embedding $E \subset Y$ is continuous. Also let $F \subset L_{p}(0, T ; X), 1 \leq p \leq \infty$. We assume that for any $f \in F$ its generalized derivative belongs to $L_{r}(0, T ; Y), 1 \leq r \leq \infty$. Now let:

- $\quad F$ is bounded in $L_{p}(0, T ; X)$;

- $\left\{f^{\prime}: f \in F\right\}$ is bounded in $L_{r}(0, T ; Y)$.

Then for $p<\infty$ the set $F$ is relatively compact in $L_{p}(0, T ; E)$, and for $p=\infty$ and $r>1$ the set $F$ is relatively compact in $C([0, T] ; E)$.

Consider the set $F=\left\{v \in L_{4}\left(0, T ; V^{3}\right), v^{\prime} \in L_{2}\left(0, T ; L_{2}(\Omega)\right)\right\}$. Since the embedding $V^{3} \subset L_{4}(\Omega)$ is compact, the embedding $F \subset L_{4}\left(0, T ; L_{4}(\Omega)\right)$ is also compact.

From continuity of embeddings

$$
C\left([0, T] ; V^{3}\right) \subset L_{4}\left(0, T ; V^{3}\right), \quad L_{2}\left(0, T ; V^{3}\right) \subset L_{2}\left(0, T ; L_{2}(\Omega)\right)
$$

the continuous embedding $W_{2} \subset F$ follows. In addition, also we have that the operator $B$ : $L_{4}\left(0, T ; L_{4}(\Omega)\right) \rightarrow L_{2}\left(0, T ; V^{-1}\right)$ is continuous (from the second item of this lemma). Thus, we have the superposition of embeddings:

$$
W_{2} \subset F \subset L_{4}\left(0, T ; L_{4}(\Omega)\right) \stackrel{B}{\rightarrow} L_{2}\left(0, T ; V^{-1}\right),
$$


where the first embedding is continuous, the second is compact, and the map $B$ is continuous. Therefore, for any function $v \in W_{2}$ we obtain that the function $B(v) \in L_{2}\left(0, T ; V^{-1}\right)$, and the map $B: W_{2} \rightarrow L_{2}\left(0, T ; V^{-1}\right)$ is compact. The proof is complete.

We proceed to investigate the properties of the map $C$. We introduce the following norm $\|v\|_{k, L_{2}\left(0, T ; V^{-1}\right)}$ equal to the norm $\|\bar{v}\|_{L_{2}\left(0, T ; V^{-1}\right)}$ where $\bar{v}(t)=e^{-k t} v(t), k \geq 0$. Then the following lemma holds.

Lemma 3. For any $v \in L_{2}\left(0, T ; V^{1}\right), z \in C G$ we have that $C(v, z) \in L_{2}\left(0, T ; V^{-1}\right)$ and the map $C$ : $L_{2}\left(0, T ; V^{1}\right) \times C G \rightarrow L_{2}\left(0, T ; V^{-1}\right)$ is continuous and bounded. In addition, for any fixed function $z \in C G$ and arbitrary $u, v \in L_{2}\left(0, T ; V^{1}\right)$ the following estimate holds:

$$
\|C(v, z)-C(u, z)\|_{k, L_{2}\left(0, T ; V^{-1}\right)} \leq C_{9} T^{1-\beta \sqrt{T / k}}\|v-u\|_{k, L_{2}\left(0, T ; V^{1}\right)} .
$$

Proof. The first part of this lemma is proved similarly to the Lemma 2.2 [30]. We prove necessary estimate (25). Let $\bar{v}(t)=e^{-k t} v(t), \bar{u}(t)=e^{-k t} u(t)$. By definition, for almost all $t \in[0, T]$ we have $\varphi \in L_{2}\left(0, T, V^{1}\right)$ and obtain

$$
\begin{aligned}
\left\langle e^{-k t} C(v, z)(t)-e^{-k t} C(u, z)\right. & (t), \varphi(t)\rangle \\
& =\int_{0}^{T} \int_{\Omega} \int_{0}^{t} e^{-k(t-s)}(t-s)^{-\beta} \mathcal{E}_{i j}(\bar{v}-\bar{u})(s, z(s ; t, x)) d s \mathcal{E}_{i j}(\varphi)(t) d x d t .
\end{aligned}
$$

Then, using the Holder inequality, we obtain

$$
\begin{gathered}
\left\langle e^{-k t} C(v, z)(t)-e^{-k t} C(u, z)(t), \varphi(t)\right\rangle \leq \int_{0}^{T} \int_{0}^{t} e^{-k(t-s)}(t-s)^{-\beta}\left(\int_{\Omega} \mathcal{E}^{2}(\bar{v}-\bar{u})(s, z(s ; t, x)) d x\right)^{1 / 2} \\
\times\left(\int_{\Omega} \mathcal{E}^{2}(\varphi)(t, x) d x\right)^{1 / 2} d s d t=\int_{0}^{T} \int_{0}^{t} e^{-k(t-s)}(t-s)^{-\beta}\|(\bar{v}-\bar{u})(s, \cdot)\|_{V^{1}}\|\varphi(t, \cdot)\|_{V^{1}} d s d t \\
\leq C_{9} T^{1-\beta}\|\bar{v}-\bar{u}\|_{L_{2}\left(0, T ; V^{1}\right)}\|\varphi\|_{L_{2}\left(0, T ; V^{1}\right)}\left(\int_{0}^{T} \int_{0}^{t} e^{-k(t-s)} d s d t\right)^{1 / 2} .
\end{gathered}
$$

The last inequality holds by virtue of the following estimate [41] (Theorem 2.6)

$$
\left\|\int_{0}^{t}(t-s)^{-\beta} \varphi(s) d s\right\|_{L_{p}(0, T)} \leq C_{9} T^{1-\beta}\|\varphi(s)\|_{L_{p}(0, T)}, \varphi(s) \in L_{p}(0, T), 1 \leq p<\infty .
$$

Estimate the remaining integral:

$$
\left(\int_{0}^{T} \int_{0}^{t} e^{-k(t-s)} d s d t\right)^{1 / 2}=\frac{1}{k} \int_{0}^{T} 1-e^{-k t} d t \leq \frac{1}{k} \int_{0}^{T} d t=\frac{T}{k}
$$

Thus, we obtain the estimate

$$
\left\langle e^{-k t} C(v, z)(t)-e^{-k t} C(u, z)(t), \varphi(t)\right\rangle \leq C_{9} T^{1-\beta \sqrt{T / k}}\|\bar{v}-\bar{u}\|_{L_{2}\left(0, T ; V^{1}\right)}\|\varphi\|_{L_{2}\left(0, T ; V^{1}\right)} .
$$

From where necessary estimate (25) follows.

We formulate one more necessary property of the operator $C$.

But first we define several concepts concerning the measure of noncompactness and $L$-condensing operators [30,42].

Definition 4. A non-negative real function $\psi$ defined on a subset of a Banach space $F$ is called a measure of non-compactness if for any subset $\mathcal{M}$ of this space the following properties are satisfied: 
- $\quad \psi(\overline{c o} \mathcal{M})=\psi(\mathcal{M})$

- for any two sets $\mathcal{M}_{1}$ and $\mathcal{M}_{2}$ from $\mathcal{M}_{1} \subset \mathcal{M}_{2}$ follows that $\psi\left(\mathcal{M}_{1}\right) \leq \psi\left(\mathcal{M}_{2}\right)$.

Here, by $\overline{c o} \mathcal{M}$ we denote the convex closure of the set $\mathcal{M}$. As an example of a measure of non-compactness, we give the Kuratowski measure of non-compactness: the exact lower bound $d>0$ for which the set $\mathcal{M}$ can be divided into a finite number of subsets whose diameters are less than $d$. Kuratowski's non-compactness measure has several important properties:

- $\quad \psi(\mathcal{M})=0$, if $\mathcal{M}$ is a relatively compact subset;

- $\psi(\mathcal{M} \cup K)=\psi(\mathcal{M})$ if $K$ is a relatively compact set.

Definition 5. Let $X$ be bounded subset of a Banach space, and $L: X \rightarrow F$ is a map from $X$ into a Banach space $F$. A map $g: X \rightarrow F$ is called L-condensing if $\psi(g(\mathcal{M}))<\psi(L(\mathcal{M}))$ for any set $\mathcal{M} \subseteq X$ such that $\psi(g(\mathcal{M})) \neq 0$.

Let $\gamma_{k}$ be the Kuratowski measure of noncompactness in the space $L_{2}\left(0, T ; V^{-1}\right)$ with the norm $\|v\|_{k, L_{2}\left(0, T ; V^{-1}\right)}$. Then the following lemma holds.

Lemma 4. The map $G: W_{2} \rightarrow L_{2}\left(0, T ; V^{-1}\right) \times V^{3}$ is L-condensing with respect to the Kuratowski measure of noncompactness $\gamma_{k}$.

Proof. Let $M \subset W_{2} \subset L_{2}\left(0, T ; V^{1}\right)$ be an arbitrary bounded set. By virtue of Theorem 2, the set $z(M)$ is the set of trajectories $z$ that are uniquely determined by the velocities $v \in M$ and this set is relatively compact. Then for any fixed $v \in W_{2}$ the set $C(v, z(M))$ is relatively compact. In addition, for any $z \in z(M)$, the map $C(\cdot, z)$ satisfies the Lipschitz condition with constant $C_{9} T^{1-\beta} \sqrt{T / k}$ in the norms $\|\cdot\|_{k, L_{2}\left(0, T, V^{1}\right)}$ and $\|\cdot\|_{k, L_{2}\left(0, T, V^{-1}\right)}$. Then, by Theorem 1.5.7 [42], the map $C(v, z)$ and, therefore, the map $G$ are $C_{9} T^{1-\beta} \sqrt{T / k}$-bounded with respect to the Hausdorff measure $\chi_{k}$. It is known, see Theorem 1.1.7 [42], that the non-compactness measures of Hausdorff and Kuratowski satisfy the following inequalities $\chi_{k}(M) \leq \gamma_{k}(M) \leq 2 \chi_{k}(M)$. Therefore, the estimate

$$
\gamma_{k}(G(M)) \leq C_{9} T^{1-\beta} \sqrt{T / k} \gamma_{k}(L(M))
$$

hold. Choosing $k$ so that $C_{9} T^{1-\beta} \sqrt{T / k}<1$, we obtain the statement of the lemma.

Using the above estimates and the properties of the operators, we prove the following a priori estimates for auxiliary family (10)-(14), (8).

Lemma 5. Let $v_{0} \in V^{3}$. Then for any solution $v \in W_{2}$ of operator inclusion (16) the following estimates hold:

$$
\begin{aligned}
& \|v\|_{L_{2}\left(0, T ; V^{1}\right)} \leq C_{10}\left(\left\|v_{0}\right\|_{V^{0}}+\sqrt{\varepsilon}\left\|v_{0}\right\|_{V^{2}}\right) ; \\
& \|v\|_{C\left([0, T] ; V^{0}\right)} \leq C_{11}\left(\left\|v_{0}\right\|_{V^{0}}+\sqrt{\varepsilon}\left\|v_{0}\right\|_{V^{2}}\right) ; \\
& \varepsilon\|v\|_{C\left([0, T] ; V^{2}\right)}^{2} \leq C_{12}\left(\left\|v_{0}\right\|_{V^{0}}^{2}+\varepsilon\left\|v_{0}\right\|_{V^{2}}^{2}\right),
\end{aligned}
$$

where the constants $C_{10}, C_{11}, C_{12}$ do not depend on $\varepsilon$ and $\xi$. 
Proof. Let $v \in W_{2}$ be a solution of operator inclusion (16). Then for any $\varphi \in V^{1}$ and almost all $t \in(0, T)$ equality (15) holds. Since it is valid for all $\varphi \in V^{1}$, we assume that $\varphi=\bar{v}$, where $\bar{v}(t)=e^{-k t} v(t)$. Then

$$
\begin{aligned}
& \int_{\Omega} v^{\prime}(t) \bar{v}(t) d x-\xi \int_{\Omega} \sum_{i, j=1}^{n}\left(\Delta_{\alpha}^{-1} v\right)_{i}(t) v_{j}(t) \frac{\partial \bar{v}_{j}(t)}{\partial x_{i}} d x \\
&+\frac{\mu_{1} \xi}{\Gamma(1-\beta)}\left(\int_{0}^{t}(t-s)^{-\beta} \mathcal{E}(v)(s, z(s ; t, x)) d s, \mathcal{E}(\bar{v}(t))\right) \\
& \quad-\varepsilon \int_{\Omega} \nabla \Delta v^{\prime}(t): \nabla \bar{v}(t) d x=\xi\langle f(t), \bar{v}(t)\rangle .
\end{aligned}
$$

Let us replace $v(t)=e^{k t} \bar{v}(t)$ and separately transform the terms in the left side of the last equality as follows. Consider the first term:

$$
\begin{aligned}
\int_{\Omega} v^{\prime}(t) \bar{v}(t) d x=\int_{\Omega}\left(e^{k t} \bar{v}(t)\right)^{\prime} \bar{v}(t) d x=e^{k t} \int_{\Omega} \bar{v}^{\prime}(t) \bar{v}(t) d x+k e^{k t} \int_{\Omega} \bar{v}(t) \bar{v}(t) d x \\
\quad=\frac{e^{k t}}{2} \int_{\Omega} \frac{\partial(\bar{v}(t) \bar{v}(t))}{\partial t} d x+k e^{k t}\|\bar{v}(t)\|_{V^{0}}^{2}=\frac{e^{k t}}{2} \frac{d}{d t}\|\bar{v}(t)\|_{V^{0}}^{2}+k e^{k t}\|\bar{v}(t)\|_{V^{0}}^{2} .
\end{aligned}
$$

Now we turn to the consideration of the following term:

$$
\begin{gathered}
\int_{\Omega} \sum_{i, j=1}^{n}\left(\Delta_{\alpha}^{-1} v\right)_{i}(t) v_{j}(t) \frac{\partial \bar{v}_{j}(t)}{\partial x_{i}} d x=e^{k t} \int_{\Omega} \sum_{i, j=1}^{n}\left(\Delta_{\alpha}^{-1} v\right)_{i}(t) \bar{v}_{j}(t) \frac{\partial \bar{v}_{j}(t)}{\partial x_{i}} d x \\
=\frac{e^{k t}}{2} \int_{\Omega} \sum_{i, j=1}^{n}\left(\Delta_{\alpha}^{-1} v\right)_{i}(t) \frac{\partial\left(\bar{v}_{j}(t) \bar{v}_{j}(t)\right)}{\partial x_{i}} d x=-\frac{e^{k t}}{2} \int_{\Omega} \sum_{i, j=1}^{n} \frac{\partial\left(\Delta_{\alpha}^{-1} v\right)_{i}(t)}{\partial x_{i}} \bar{v}_{j}^{2}(t) d x \\
=-\frac{e^{k t}}{2} \int_{\Omega} \sum_{j=1}^{n} \operatorname{div} u(t) \bar{v}_{j}^{2}(t) d x=0 .
\end{gathered}
$$

Finally, we transform the last term:

$$
\begin{gathered}
-\varepsilon \int_{\Omega} \nabla \Delta v^{\prime}(t): \nabla \bar{v}(t) d x=-\varepsilon \int_{\Omega} \nabla \Delta\left(e^{k t} \bar{v}(t)\right)^{\prime}: \nabla \bar{v}(t) d x-\varepsilon k e^{k t} \int_{\Omega} \nabla \Delta \bar{v}(t): \nabla \bar{v}(t) d x \\
-\varepsilon e^{k t} \int_{\Omega} \nabla \Delta \bar{v}^{\prime}(t): \nabla \bar{v}(t) d x=\varepsilon k e^{k t} \int_{\Omega} \Delta \bar{v}(t) \Delta \bar{v}(t) d x+\frac{\varepsilon e^{k t}}{2} \int_{\Omega} \frac{\partial}{\partial t}(\Delta \bar{v}(t) \Delta \bar{v}(t)) d x \\
=\varepsilon k e^{k t}\|\bar{v}(t)\|_{V^{2}}^{2}+\frac{\varepsilon e^{k t}}{2} \frac{d}{d t}\|\bar{v}(t)\|_{V^{2}}^{2} .
\end{gathered}
$$

Thus, equality (29) can be rewritten as follows:

$$
\begin{aligned}
& \frac{e^{k t}}{2} \frac{d}{d t}\|\bar{v}(t)\|_{V^{0}}^{2}+k e^{k t}\|\bar{v}(t)\|_{V^{0}}^{2}+\mu_{0} e^{k t}\|\bar{v}(t)\|_{V^{1}}^{2}+\varepsilon k e^{k t}\|\bar{v}(t)\|_{V^{2}}^{2}+\frac{\varepsilon e^{k t}}{2} \frac{d}{d t}\|\bar{v}(t)\|_{V^{2}}^{2} \\
& \quad=-\frac{\mu_{1} \xi}{\Gamma(1-\beta)}\left(\int_{0}^{t}(t-s)^{-\beta} \mathcal{E}\left(e^{k t} \bar{v}\right)(s, z(s ; t, x)) d s, \mathcal{E}(\bar{v}(t))\right)+\xi e^{k t}\langle f(t), \bar{v}(t)\rangle .
\end{aligned}
$$

We estimate modulo the right-hand side of the resulting equality. Using the Cauchy inequality

$$
b c \leq \frac{\delta b^{2}}{2}+\frac{c^{2}}{2 \delta}
$$

for $\delta=1 / \mu_{0}$, we obtain:

$$
\xi e^{k t}\langle f(t), \bar{v}(t)\rangle \leq e^{k t}\|f(t)\|_{V^{-1}}\|\bar{v}(t)\|_{V^{1}} \leq \frac{e^{k t}}{2 \mu_{0}}\|f(t)\|_{V^{-1}}^{2}+\frac{\mu_{0} e^{k t}}{2}\|\bar{v}(t)\|_{V^{1}}^{2} .
$$


Multiplying both sides of equality (31) on $e^{-k t}$, for almost all $t \in(0, T)$ we have

$$
\begin{gathered}
\frac{1}{2} \frac{d}{d t}\|\bar{v}(t)\|_{V^{0}}^{2}+\frac{\varepsilon}{2} \frac{d}{d t}\|\bar{v}(t)\|_{V^{2}}^{2}+k\|\bar{v}(t)\|_{V^{0}}^{2}+\frac{\mu_{0}}{2}\|\bar{v}(t)\|_{V^{1}}^{2}+\varepsilon k\|\bar{v}(t)\|_{V^{2}}^{2} \\
\leq \frac{\mu_{1}}{\Gamma(1-\beta)}\left|\left(e^{-k t} \int_{0}^{t}(t-s)^{-\beta} \mathcal{E}\left(e^{k t} \bar{v}\right)(s, z(s ; t, x)) d s, \mathcal{E}(\bar{v}(t))\right)\right|+\frac{1}{2 \mu_{0}}\|f(t)\|_{V^{-1}}^{2} .
\end{gathered}
$$

We integrate the last inequality with respect to $t$ from 0 to $\tau$, where $\tau \in[0, T]$. Then

$$
\begin{aligned}
& \frac{1}{2}\|\bar{v}(t)\|_{V^{0}}^{2}+\frac{\varepsilon}{2}\|\bar{v}(t)\|_{V^{2}}^{2}+k \int_{0}^{\tau}\|\bar{v}(t)\|_{V^{0}}^{2} d t+\varepsilon k \int_{0}^{\tau}\|\bar{v}(t)\|_{V^{2}}^{2} d t \\
+ & \frac{\mu_{0}}{2} \int_{0}^{\tau}\|\bar{v}(t)\|_{V^{1}}^{2} d t \leq \frac{1}{2}\left\|v_{0}\right\|_{V^{0}}^{2}+\frac{\varepsilon}{2}\left\|v_{0}\right\|_{V^{2}}^{2}+\frac{1}{2 \mu_{0}} \int_{0}^{\tau}\|f(t)\|_{V^{-1}}^{2} d t \\
+ & \frac{\mu_{1}}{\Gamma(1-\beta)} \int_{0}^{\tau}\left|\left(e^{-k t} \int_{0}^{t}(t-s)^{-\beta} \mathcal{E}\left(e^{k t} \bar{v}\right)(s, z(s ; t, x)) d s, \mathcal{E}(\bar{v}(t))\right)\right| d t .
\end{aligned}
$$

We use estimate (25) for $u=0$. In this way,

$$
\begin{gathered}
\frac{1}{2}\|\bar{v}(t)\|_{V^{0}}^{2}+\frac{\varepsilon}{2}\|\bar{v}(t)\|_{V^{2}}^{2}+k \int_{0}^{\tau}\|\bar{v}(t)\|_{V^{0}}^{2} d t+\varepsilon k \int_{0}^{\tau}\|\bar{v}(t)\|_{V^{2}}^{2} d t \\
+\frac{\mu_{0}}{2} \int_{0}^{\tau}\|\bar{v}(t)\|_{V^{1}}^{2} d t \leq \frac{1}{2}\left\|v_{0}\right\|_{V^{0}}^{2}+\frac{\varepsilon}{2}\left\|v_{0}\right\|_{V^{2}}^{2}+\frac{\mu_{1} C_{9} T^{1-\beta} \sqrt{T /(2 k)}}{\Gamma(1-\beta)}\|\bar{v}\|_{L_{2}\left(0, T ; V^{1}\right)}^{2}+\frac{1}{2 \mu_{0}}\|f\|_{L_{2}\left(0, T ; V^{-1}\right)}^{2} .
\end{gathered}
$$

We assume that the number $k$ is sufficiently large such that $\frac{\mu_{1} C_{9} T^{1-\beta} \sqrt{T /(2 k)}}{\Gamma(1-\beta)} \leq \mu_{0} / 4$. The nonnegativity of the quantities $\|\bar{v}(t)\|_{V^{0}}^{2}\|\bar{v}(t)\|_{V^{2}}^{2}$ and $\|\bar{v}(t)\|_{V^{1}}^{2}$ yields the following estimates:

$$
\begin{gathered}
\frac{\mu_{0}}{2} \int_{0}^{\tau}\|\bar{v}(t)\|_{V^{1}}^{2} d t \leq \frac{1}{2}\left\|v_{0}\right\|_{V^{0}}^{2}+\frac{\varepsilon}{2}\left\|v_{0}\right\|_{V^{2}}^{2}+\frac{1}{2 \mu_{0}}\|f\|_{L_{2}\left(0, T ; V^{-1}\right)}^{2}+\frac{\mu_{0}}{4}\|\bar{v}\|_{L_{2}\left(0, T ; V^{1}\right)^{\prime}}^{2} \\
\frac{\varepsilon}{2}\|\bar{v}(t)\|_{V^{2}}^{2} \leq \frac{1}{2}\left\|v_{0}\right\|_{V^{0}}^{2}+\frac{\varepsilon}{2}\left\|v_{0}\right\|_{V^{2}}^{2}+\frac{1}{2 \mu_{0}}\|f\|_{L_{2}\left(0, T ; V^{-1}\right)}^{2}+\frac{\mu_{0}}{4}\|\bar{v}\|_{L_{2}\left(0, T ; V^{1}\right)^{\prime}}^{2} \\
\frac{1}{2}\|\bar{v}(t)\|_{V^{0}}^{2} \leq \frac{1}{2}\left\|v_{0}\right\|_{V^{0}}^{2}+\frac{\varepsilon}{2}\left\|v_{0}\right\|_{V^{2}}^{2}+\frac{1}{2 \mu_{0}}\|f\|_{L_{2}\left(0, T ; V^{-1}\right)}^{2}+\frac{\mu_{0}}{4}\|\bar{v}\|_{L_{2}\left(0, T ; V^{1}\right)^{2}}^{2} .
\end{gathered}
$$

Since the right-hand side in all the above inequalities does not depend on $\tau$, we pass to the maximum in $\tau \in[0, T]$ in the left-hand sides of these inequalities. Then

$$
\begin{aligned}
& \frac{\mu_{0}}{2}\|\bar{v}\|_{L_{2}\left(0, T ; V^{1}\right)}^{2} \leq \frac{1}{2}\left\|v_{0}\right\|_{V^{0}}^{2}+\frac{\varepsilon}{2}\left\|v_{0}\right\|_{V^{2}}^{2}+\frac{1}{2 \mu_{0}}\|f\|_{L_{2}\left(0, T ; V^{-1}\right)}^{2}+\frac{\mu_{0}}{4}\|\bar{v}\|_{L_{2}\left(0, T ; V^{1}\right)^{\prime}}^{2} \\
& \frac{\varepsilon}{2}\|\bar{v}\|_{C\left([0, T] ; V^{2}\right)}^{2} \leq \frac{1}{2}\left\|v_{0}\right\|_{V^{0}}^{2}+\frac{\varepsilon}{2}\left\|v_{0}\right\|_{V^{2}}^{2}+\frac{1}{2 \mu_{0}}\|f\|_{L_{2}\left(0, T ; V^{-1}\right)}^{2}+\frac{\mu_{0}}{4}\|\bar{v}\|_{L_{2}\left(0, T ; V^{1}\right)^{\prime}}^{2} \\
& \frac{1}{2}\|\bar{v}\|_{C\left([0, T] ; V^{0}\right)}^{2} \leq \frac{1}{2}\left\|v_{0}\right\|_{V^{0}}^{2}+\frac{\varepsilon}{2}\left\|v_{0}\right\|_{V^{2}}^{2}+\frac{1}{2 \mu_{0}}\|f\|_{L_{2}\left(0, T ; V^{-1}\right)}^{2}+\frac{\mu_{0}}{4}\|\bar{v}\|_{L_{2}\left(0, T ; V^{1}\right)}^{2} .
\end{aligned}
$$

From this and feedback condition (8) the required estimates (26)-(28) directly follow. The proof is complete. 
Lemma 6. Let $v_{0} \in V^{3}$. For any solution $v \in W_{2}$ for operator inclusion (16) we have the following estimates

$$
\begin{gathered}
\varepsilon\left\|v^{\prime}\right\|_{L_{2}\left(0, T ; V^{3}\right)} \leq C_{13}\left(1+\frac{1}{\varepsilon}\right)\left\|v_{0}\right\|_{V^{0}}^{2}+C_{13} \sqrt{\varepsilon}\left\|v_{0}\right\|_{V^{2}}+C_{13}\left\|v_{0}\right\|_{V^{2}}^{2} \\
\|v\|_{C\left([0, T] ; V^{3}\right)} \leq\left\|v_{0}\right\|_{V^{3}}+\frac{C_{13} T^{\frac{1}{2}}}{\varepsilon}\left(1+\frac{1}{\varepsilon}\right)\left\|v_{0}\right\|_{V^{0}}^{2}+\frac{C_{13} T^{\frac{1}{2}}}{\sqrt{\varepsilon}}\left\|v_{0}\right\|_{V^{2}}+\frac{C_{13} T^{\frac{1}{2}}}{\varepsilon}\left\|v_{0}\right\|_{V^{2}}^{2} \\
\left\|v^{\prime}\right\|_{L_{4 / 3}\left(0, T ; V^{-1}\right)} \leq C_{14}\left(\left\|v_{0}\right\|_{V^{0}}^{2}+\varepsilon\left\|v_{0}\right\|_{V^{2}}^{2}+1\right) \\
\varepsilon\left\|v^{\prime}\right\|_{L_{4 / 3}\left(0, T ; V^{3}\right)} \leq C_{15}\left(\left\|v_{0}\right\|_{V^{0}}^{2}+\varepsilon\left\|v_{0}\right\|_{V^{2}}^{2}+1\right)
\end{gathered}
$$

where the constants $C_{13}, C_{14}, C_{15}$ do not depend on $\varepsilon, v$ and $\xi$.

Proof. Let $v \in W_{2}$ be a solution of (16). Then it satisfies the following operator equality

$$
J v^{\prime}+\varepsilon A_{2} v^{\prime}+\mu_{0} A v-\xi B(v)+\frac{\mu_{1} \xi}{\Gamma(1-\beta)} C(v, z)=\xi f .
$$

Hence,

$$
\left\|\left(J+\varepsilon A_{2}\right) v^{\prime}\right\|_{L_{2}\left(0, T ; V^{-1}\right)}=\left\|\xi f+\xi B(v)-\mu_{0} A v-\frac{\mu_{1} \xi}{\Gamma(1-\beta)} C(v, z)\right\|_{L_{2}\left(0, T ; V^{-1}\right)} .
$$

We estimate the right-hand side of the last equality. By estimates (18) and (25) for $u=0$, we get:

$$
\begin{aligned}
\| \xi f+\xi B(v)- & \mu_{0} A v-\frac{\mu_{1} \xi}{\Gamma(1-\beta)} C(v, z) \|_{L_{2}\left(0, T ; V^{-1}\right)} \\
& \leq\|f\|_{L_{2}\left(0, T ; V^{-1}\right)}+\|B(v)\|_{L_{2}\left(0, T ; V^{-1}\right)}+\frac{\mu_{1} C_{9} T^{1-\beta}}{\Gamma(1-\beta)}\|v\|_{L_{2}\left(0, T ; V^{1}\right)}+\mu_{0}\|v\|_{L_{2}\left(0, T ; V^{1}\right)} .
\end{aligned}
$$

We separately estimate the $\|B(v)\|_{L_{2}\left(0, T ; V^{-1}\right)}$. Using (23), and the continuity of the embedding $V^{2} \subset L_{4}(\Omega)$, we have:

$$
\begin{aligned}
\|B(v)\|_{L_{2}\left(0, T ; V^{-1}\right)}=\left(\int_{0}^{T}\|B(v)\|_{V^{-1}}^{2} d t\right)^{\frac{1}{2}} \leq C_{3}\left(\int_{0}^{T}\|v(t)\|_{L_{4}(\Omega)}^{4} d t\right)^{\frac{1}{2}} \\
\quad \leq C_{16}\left(\int_{0}^{T}\|v(t)\|_{V^{2}}^{4} d t\right)^{\frac{1}{2}} \leq C_{16} T^{\frac{1}{2}} \max _{t \in[0, T]}\|v(t)\|_{V^{2}}^{2}=C_{16} T^{\frac{1}{2}}\|v\|_{C\left([0, T] ; V^{2}\right)}^{2}
\end{aligned}
$$

We rewrite inequality (37) as follows

$$
\begin{aligned}
& \left\|\xi f+\xi B(v)-\mu_{0} A v-\frac{\mu_{1} \xi}{\Gamma(1-\beta)} C(v, z)\right\|_{L_{2}\left(0, T ; V^{-1}\right)} \\
& \quad \leq C_{17}\left(\|f\|_{L_{2}\left(0, T ; V^{-1}\right)}+C_{16} T^{1 / 2}\|v\|_{C\left([0, T] ; V^{2}\right)}^{2}+\|v\|_{L_{2}\left(0, T ; V^{1}\right)}\right) .
\end{aligned}
$$

From the a priori estimates (26) and (28) it immediately follows that

$$
\left\|\left(J+\varepsilon A_{2}\right) v^{\prime}\right\|_{L_{2}\left(0, T ; V^{-1}\right)} \leq C_{13}\left(1+\frac{1}{\varepsilon}\right)\left\|v_{0}\right\|_{V^{0}}^{2}+C_{13} \sqrt{\varepsilon}\left\|v_{0}\right\|_{V^{2}}+C_{13}\left\|v_{0}\right\|_{V^{2}}^{2} .
$$

To prove estimate (32), it remains to use the left (21) for $p=2$ :

$$
\begin{aligned}
\varepsilon\left\|v^{\prime}\right\|_{L_{2}\left(0, T ; V^{3}\right)} \leq \|\left(J+\varepsilon A_{2}\right) & v^{\prime} \|_{L_{2}\left(0, T ; V^{-1}\right)} \\
& \leq C_{13}\left(1+\frac{1}{\varepsilon}\right)\left(\left\|v_{0}\right\|_{V^{0}}^{2}+\|f\|_{L_{2}\left(0, T ; V^{-1}\right)}^{2}\right)+C_{13} \sqrt{\varepsilon}\left\|v_{0}\right\|_{V^{2}}+C_{13}\left\|v_{0}\right\|_{V^{2}}^{2} .
\end{aligned}
$$

Hence, inequality (32) is established. 
We pass to the proof of estimate (33). Represent the function $v \in W_{2}$ as follows:

$$
v(t)=\int_{0}^{t} v^{\prime}(s) d s+v_{0} .
$$

Then

$$
\|v(t)\|_{V^{3}} \leq\left\|v_{0}+\int_{0}^{t} v^{\prime}(s) d s\right\|_{V^{3}} \leq\left\|v_{0}\right\|_{V^{3}}+\int_{0}^{t}\left\|v^{\prime}(s)\right\|_{V^{3}} d s \leq\left\|v_{0}\right\|_{V^{3}}+T^{\frac{1}{2}}\left\|v^{\prime}\right\|_{L_{2}\left(0, T ; V^{3}\right)} .
$$

Since the right-hand side of the resulting inequality does not depend on $t$, we pass to the maximum in $\tau \in[0, T]$ in the left-hand side. Then, taking into account estimate (32), we obtain

$$
\max _{t \in[0, T]}\|v(t)\|_{V^{3}} \leq\left\|v_{0}\right\|_{V^{3}}+\frac{C_{13} T^{\frac{1}{2}}}{\varepsilon}\left(1+\frac{1}{\varepsilon}\right)\left\|v_{0}\right\|_{V^{0}}^{2}+\frac{C_{13} T^{\frac{1}{2}}}{\sqrt{\varepsilon}}\left\|v_{0}\right\|_{V^{2}}+\frac{C_{13} T^{\frac{1}{2}}}{\varepsilon}\left\|v_{0}\right\|_{V^{2}}^{2} .
$$

Thus, we received estimate (33).

Now we prove inequality (34). As before, $v \in W_{2}$ is a solution of operator Equation (36). Then

$$
\begin{aligned}
\left\|v^{\prime}\right\|_{L_{4 / 3}\left(0, T ; V^{-1}\right) \leq} & \left\|\xi f+\xi B(v)-\mu_{0} A v-\frac{\mu_{1} \xi}{\Gamma(1-\beta)} C(v, z)-\varepsilon A^{2} v^{\prime}\right\|_{L_{4 / 3}\left(0, T ; V^{-1}\right)} \\
\leq\|f\|_{L_{4 / 3}\left(0, T ; V^{-1}\right)}+ & \|B(v)\|_{L_{4 / 3}\left(0, T ; V^{-1}\right)}+\mu_{0}\|A v\|_{L_{4 / 3}\left(0, T ; V^{-1}\right)} \\
& +\frac{\mu_{1}}{\Gamma(1-\beta)}\|C(v, z)\|_{L_{4 / 3}\left(0, T ; V^{-1}\right)}+\varepsilon\left\|A^{2} v^{\prime}\right\|_{L_{4 / 3}\left(0, T ; V^{-1}\right)}
\end{aligned}
$$

We separately consider the terms on the right-hand side of the last inequality. First, we estimate $\|B(v)\|_{L_{4 / 3}\left(0, T ; V^{-1}\right)}$. Given from Reference [34] the well-known inequality for $n=3$

$$
\|u\|_{L_{4}(\Omega)} \leq 2^{\frac{1}{2}}\|u\|_{L_{2}(\Omega)}^{\frac{1}{4}}\|\nabla u\|_{L_{2}(\Omega)^{\prime}}^{\frac{3}{4}} \quad u \in V^{1},
$$

and estimate (23), we obtain (for the case $n=2$ the proof is similar):

$$
\begin{gathered}
\|B(v)\|_{L_{4 / 3}\left(0, T ; V^{-1}\right)}=\left(\int_{0}^{T}\|B(v)\|_{V^{-1}}^{\frac{4}{3}} d t\right)^{\frac{3}{4}} \leq C_{3}\left(\int_{0}^{T}\|v\|_{L_{4}(\Omega)}^{\frac{8}{3}} d t\right)^{\frac{3}{4}} \\
\leq 2 C_{3}\left(\int_{0}^{T}\|v\|_{L_{2}(\Omega)}^{\frac{2}{3}}\|\nabla v\|_{L_{2}(\Omega)}^{2} d t\right)^{\frac{3}{4}} \leq C_{18}\left(\int_{0}^{T}\|v\|_{V^{0}}^{\frac{2}{3}}\|v\|_{V^{1}}^{2} d t\right)^{\frac{3}{4}} \\
\leq C_{18}\|v\|_{C\left([0, T] ; V^{0}\right)}^{\frac{1}{2}}\left(\int_{0}^{T}\|v\|_{V^{1}}^{2} d t\right)^{\frac{3}{4}}=C_{18}\|v\|_{C\left([0, T] ; V^{0}\right)}^{\frac{1}{2}}\|v\|_{L_{2}\left(0, T ; V^{1}\right)}^{\frac{3}{2}} .
\end{gathered}
$$

Consider the following term. We use the Holder inequality and estimate (18). Then

$$
\begin{aligned}
\|A v\|_{L_{4 / 3}\left(0, T ; V^{-1}\right)}=\left(\int_{0}^{T}\|A v\|_{V^{-1}}^{\frac{4}{3}} d t\right)^{\frac{3}{4}} \leq\left(\int_{0}^{T}\|v\|_{V^{1}}^{\frac{4}{3}} d t\right)^{\frac{3}{4}} & \\
& \leq T^{\frac{1}{4}}\left(\int_{0}^{T}\|v\|_{V^{1}}^{2} d t\right)^{\frac{1}{2}}=T^{\frac{1}{4}}\|v\|_{L_{2}\left(0, T ; V^{1}\right)} .
\end{aligned}
$$

Similarly, using the Holder inequality and estimate (25) for $u=0$, we obtain an estimate for the next term:

$$
\begin{aligned}
\|C(v, z)\|_{L_{4 / 3}\left(0, T ; V^{-1}\right)}=\left(\int_{0}^{T}\|C(v, z)\|_{V^{-1}}^{\frac{4}{3}} d t\right)^{\frac{3}{4}} & \leq T^{\frac{1}{4}}\left(\int_{0}^{T}\|C(v, z)\|_{V^{-1}}^{2} d t\right)^{\frac{1}{2}} \\
& =T^{\frac{1}{4}}\|C(v, z)\|_{L_{2}\left(0, T ; V^{-1}\right)} \leq T^{\frac{1}{4}} T^{1-\beta} C_{9}\|v\|_{L_{2}\left(0, T ; V^{1}\right)} .
\end{aligned}
$$


Finally, we consider the last term. Using inequality (20), we get:

$$
\varepsilon\left\|A^{2} v^{\prime}\right\|_{L_{4 / 3}\left(0, T ; V^{-1}\right)}=\varepsilon\left(\int_{0}^{T}\left\|A^{2} v^{\prime}\right\|_{V^{-1}}^{\frac{4}{3}} d t\right)^{\frac{3}{4}} \leq \varepsilon\left(\int_{0}^{T}\left\|v^{\prime}\right\|_{V^{3}}^{\frac{4}{3}} d t\right)^{\frac{3}{4}} \leq \varepsilon\left\|v^{\prime}\right\|_{L_{4 / 3}\left(0, T ; V^{3}\right)} .
$$

Let us estimate the right-hand side of the last inequality. We use the left side of estimate (22) for $p=4 / 3$. Thus, to obtain an estimate of $\varepsilon\left\|v^{\prime}\right\|_{L_{4 / 3}\left(0, T ; V^{3}\right)}$, it is necessary to obtain an estimate of $\left\|\left(J+\varepsilon A_{2}\right) v^{\prime}\right\|_{L_{4 / 3}\left(0, T ; V^{-1}\right)}$. To do this, we again use operator Equation (36). From its appearance, it follows that

$\varepsilon\left\|v^{\prime}\right\|_{L_{4 / 3}\left(0, T ; V^{3}\right)} \leq\|f\|_{L_{4 / 3}\left(0, T ; V^{-1}\right)}+\|B(v)\|_{L_{4 / 3}\left(0, T ; V^{-1}\right)}-\mu_{0}\|A v\|_{L_{4 / 3}\left(0, T ; V^{-1}\right)}+\mu_{1}\|C(v, z)\|_{L_{4 / 3}\left(0, T ; V^{-1}\right)}$.

Thus,

$$
\begin{aligned}
& \varepsilon\left\|A^{2} v^{\prime}\right\|_{L_{4 / 3}\left(0, T ; V^{-1}\right)} \leq \varepsilon\left\|v^{\prime}\right\|_{L_{4 / 3}\left(0, T ; V^{3}\right)} \\
& \leq\|f\|_{L_{4 / 3}\left(0, T ; V^{-1}\right)}+\|B(v)\|_{L_{4 / 3}\left(0, T ; V^{-1}\right)}+\mu_{0}\|A v\|_{L_{4 / 3}\left(0, T ; V^{-1}\right)}+\frac{\mu_{1}}{\Gamma(1-\beta)}\|C(v, z)\|_{L_{4 / 3}\left(0, T ; V^{-1}\right)} .
\end{aligned}
$$

From (38), estimates (39)-(41) and a priori estimates (26) and (27), we get

$$
\begin{gathered}
\left\|v^{\prime}\right\|_{L_{4 / 3}\left(0, T ; V^{-1}\right)} \leq 2\left(\|f\|_{L_{4 / 3}\left(0, T ; V^{-1}\right)}+\|B(v)\|_{L_{4 / 3}\left(0, T ; V^{-1}\right)}+\mu_{0}\|A v\|_{L_{4 / 3}\left(0, T ; V^{-1}\right)}\right. \\
\left.+\frac{\mu_{1}}{\Gamma(1-\beta)}\|C(v, z)\|_{L_{4 / 3}\left(0, T ; V^{-1}\right)}\right) \leq C_{19}\left(\|f\|_{L_{2}\left(0, T ; V^{-1}\right)}+\|v\|_{L_{2}\left(0, T ; V^{1}\right)}\right. \\
\left.+\|v\|_{C\left([0, T] ; V^{0}\right)}^{\frac{1}{2}}\|v\|_{L_{2}\left(0, T ; V^{1}\right)}^{\frac{3}{2}}\right) \leq C_{20}\left(\|f\|_{L_{2}\left(0, T ; V^{-1}\right)}+\left\|v_{0}\right\|_{V^{0}}+\sqrt{\varepsilon}\left\|v_{0}\right\|_{V^{2}}\right. \\
\left.+\left(\left\|v_{0}\right\|_{V^{0}}+\sqrt{\varepsilon}\left\|v_{0}\right\|_{V^{2}}+\|f\|_{L_{2}\left(0, T ; V^{-1}\right)}\right)^{\frac{1}{2}}\left(\left\|v_{0}\right\|_{V^{0}}+\sqrt{\varepsilon}\left\|v_{0}\right\|_{V^{2}}+\|f\|_{L_{2}\left(0, T ; V^{-1}\right)}\right)\right)^{\frac{3}{2}} \\
\leq C_{21}\left(\left\|v_{0}\right\|_{V^{0}}+\sqrt{\varepsilon}\left\|v_{0}\right\|_{V^{2}}+\|f\|_{L_{2}\left(0, T ; V^{-1}\right)}+1\right)^{2} \leq 4 C_{21}\left(\left\|v_{0}\right\|_{V^{0}}^{2}+\varepsilon\left\|v_{0}\right\|_{V^{2}}^{2}+1\right) .
\end{gathered}
$$

This completes the proof of inequality (34), where $C_{14}=4 C_{21}$.

Finally, applying again estimates (39) and (40), for the right-hand side of (41), as well as a priori estimates (26) and (27), we obtain

$$
\begin{gathered}
\varepsilon\left\|v^{\prime}\right\|_{L_{4 / 3}\left(0, T ; V^{3}\right)} \leq 2\left(\|f\|_{L_{4 / 3}\left(0, T ; V^{-1}\right)}+\|B(v)\|_{L_{4 / 3}\left(0, T ; V^{-1}\right)}+\mu_{0}\|A v\|_{L_{4 / 3}\left(0, T ; V^{-1}\right)}\right. \\
\left.+\frac{\mu_{1}}{\Gamma(1-\beta)}\|C(v, z)\|_{L_{4 / 3}\left(0, T ; V^{-1}\right)}\right) \leq C_{22}\left(\|f\|_{L_{2}\left(0, T ; V^{-1}\right)}+\|v\|_{L_{2}\left(0, T ; V^{1}\right)}\right. \\
\left.+\|v\|_{C\left([0, T] ; V^{0}\right)}^{\frac{1}{2}}\|v\|_{L_{2}\left(0, T ; V^{1}\right)}^{\frac{3}{2}}\right) \leq C_{23}\left(\|f\|_{L_{2}\left(0, T ; V^{-1}\right)}+\left\|v_{0}\right\|_{V^{0}}+\sqrt{\varepsilon}\left\|v_{0}\right\|_{V^{2}}\right. \\
\left.+\left(\left\|v_{0}\right\|_{V^{0}}+\sqrt{\varepsilon}\left\|v_{0}\right\|_{V^{2}}+\|f\|_{L_{2}\left(0, T ; V^{-1}\right)}\right)^{\frac{1}{2}}\left(\left\|v_{0}\right\|_{V^{0}}+\sqrt{\varepsilon}\left\|v_{0}\right\|_{V^{2}}+\|f\|_{L_{2}\left(0, T ; V^{-1}\right)}\right)^{\frac{3}{2}}\right) \\
\leq C_{24}\left(\left\|v_{0}\right\|_{V^{0}}+\sqrt{\varepsilon}\left\|v_{0}\right\|_{V^{2}}+\|f\|_{L_{2}\left(0, T ; V^{-1}\right)}+1\right)^{2} \leq 4 C_{24}\left(\left\|v_{0}\right\|_{V^{0}}^{2}+\varepsilon\left\|v_{0}\right\|_{V^{2}}^{2}+1\right) .
\end{gathered}
$$

Thus, inequality (35), where $C_{15}=4 C_{24}$ is established. The proof is complete.

Lemma 7. Let $v_{0} \in V^{3}$. Then for any solution $v \in W_{2}$ of operator Equation (16) we have the following estimate:

$$
\|v\|_{W_{2}} \leq C_{25}
$$

where $C_{25}>0$ is a constant that depends on $\varepsilon$.

Theorem 6. Let $v_{0} \in V^{3}$. Then there is at least one solution $v \in W_{2}$ of auxiliary problem (10)-(14), (8) for $\xi=1$. 
Proof. To prove this theorem, we use the topological degree theory for multi-valued vector fields [2,43]. Consider operator inclusion (17). From Corollary 7 it follows that all solutions of inclusion (17) are in the ball $B_{R} \subset W_{2}$ of radius $R=C_{25}+1$ centered at zero. By item 4 of Lemma 1 the operator $L: W_{2} \rightarrow L_{2}\left(0, T ; V^{-1}\right) \times V^{3}$ is invertible. Then there is no solution of the family of following inclusions

$$
v \in \xi \mathcal{M}, \text { where } \xi \in[0,1],
$$

on the boundary of the same ball $B_{R}$.

By virtue of item 4 of Lemma 1 the operator $L^{-1}: L_{2}\left(0, T ; V^{-1}\right) \times V^{3} \rightarrow W_{2}$ is continuous. By the Lemmas 2 and 4 the map $(\mathcal{Y}(v)+K(v)-G(v)): W_{2} \rightarrow L_{2}\left(0, T ; V^{-1}\right) \times V^{3}$ is $L$ - condensing with respect to the Kuratowski $\gamma_{k}$ non-compactness measure. Therefore, the operator $\mathcal{M}: W_{2} \rightarrow W_{2}$ is condensing with respect to the Kuratowski $\gamma_{k}$ non-compactness measure.

Thus, the vector field $v-\xi \mathcal{M}(v)$ is non-degenerate on the boundary of the ball $B_{R}$, which means that the topological degree $\operatorname{deg}\left(I-\xi \mathcal{M}(v), B_{R}, 0\right)$ is defined for this vector field . By the properties of homotopy invariance and normalization of degree we obtain that

$$
\operatorname{deg}\left(I-\mathcal{M}(v), B_{R}, 0\right)=\operatorname{deg}\left(I, B_{R}, 0\right)=1 .
$$

The non-zero degree of the mapping ensures the existence of at least one solution $v \in W_{2}$ of inclusion (17) for $\xi=1$, and therefore of auxiliary problem (8), (10)-(14) for $\xi=1$. The theorem is proved.

\section{Proof of Theorem 3}

We proceed directly to the proof of the solvability of feedback control problem (1)-(5), (8). To do this, we carry out the passage to the limit in auxiliary problem (10)-(14), (8) for $\xi=1$. Since the space $V^{3}$ is dense in $V^{0}$, then for each $v_{0}^{*} \in V^{0}$ there exists a sequence $v_{0}^{m} \in V^{3}$ converging to $v_{0}^{*}$ in $V^{0}$. If $v_{0}^{*} \equiv 0$, then we put $v_{0}^{m} \equiv 0, \varepsilon_{m}=1 / m$. If $\left\|v_{0}^{*}\right\|_{V^{0}} \neq 0$, then starting from some number we have $\left\|v_{0}^{m}\right\|_{V^{2}} \neq 0$. Then we put $\varepsilon_{m}=1 /\left(m\left\|v_{0}^{m}\right\|_{V^{2}}^{2}\right)$. Under our choice $\left\{\varepsilon_{m}\right\}$ resulting sequence converges to zero as $m \rightarrow \infty$. Moreover, $\varepsilon_{m}\left\|v_{0}^{m}\right\|_{V^{2}}^{2} \leq 1$.

By Theorem 6, for each $\varepsilon_{m}$ and $v_{0}^{m}$ there exists a solution $v_{m} \in W_{2} \subset W_{1}$ of auxiliary problem (10)-(14), (8) for $\xi=1$. Thus, each solution $v_{m}$ for all $\varphi \in V^{1}$ for almost all $t \in(0, T)$ satisfies the equality

$$
\begin{gathered}
\left\langle v_{m}^{\prime}, \varphi\right\rangle-\int_{\Omega} \sum_{i, j=1}^{n}\left(\Delta_{\alpha}^{-1} v_{m}\right)_{i}\left(v_{m}\right)_{j} \frac{\partial \varphi_{j}}{\partial x_{i}} d x+\mu_{0} \int_{\Omega} \nabla v_{m}: \nabla \varphi d x \\
-\varepsilon_{m} \int_{\Omega} \nabla \Delta v_{m}^{\prime}: \nabla \varphi d x+\frac{\mu_{1}}{\Gamma(1-\beta)}\left(\int_{0}^{t}(t-s)^{-\beta} \mathcal{E}\left(v_{m}\right)\left(s, z_{m}(s ; t, x)\right) d s, \mathcal{E}(\varphi)\right)=\left\langle f_{m}, \varphi\right\rangle,
\end{gathered}
$$

and the initial condition

$$
\left.v_{m}\right|_{t=0}=v_{0}^{m} .
$$

Since the sequence $\left\{v_{0}^{m}\right\}$ converges in $V^{0}$, it is bounded by the norm $V^{0}$. Hence,

$$
\left\|v_{0}^{m}\right\|_{V^{0}}^{2}+\varepsilon_{m}\left\|v_{0}^{m}\right\|_{V^{2}}^{2} \leq C_{26} .
$$


Thus, from estimates (26), (27), (34) and (35) we obtain that

$$
\begin{gathered}
\left\|v_{m}\right\|_{L_{2}\left(0, T ; V^{1}\right)}^{2} \leq C_{27}, \\
\left\|v_{m}\right\|_{C\left([0, T] ; V^{0}\right)}^{2} \leq C_{28}, \\
\left\|v_{m}^{\prime}\right\|_{L_{4 / 3}\left(0, T ; V^{-1}\right)} \leq C_{29}, \\
\varepsilon\left\|v_{m}^{\prime}\right\|_{L_{4 / 3}\left(0, T ; V^{3}\right)} \leq C_{30},
\end{gathered}
$$

where the constants $C_{27}, C_{28}, C_{29}, C_{30}$ do not depend on $\varepsilon$. Due to the continuity of the embedding $C\left([0, T] ; V^{0}\right) \subset L_{\infty}\left(0, T ; V^{0}\right)$ and estimates (44)-(46), without loss of generality (if necessary, passing to a subsequence) we obtain that

$$
\begin{gathered}
v_{m} \rightarrow v_{*} \text { weakly in } L_{2}\left(0, T ; V^{1}\right) \text { as } m \rightarrow \infty, \\
v_{m} \rightarrow v_{*} \text { *-weakly in } L_{\infty}\left(0, T ; V^{0}\right) \text { as } m \rightarrow \infty, \\
v_{m}^{\prime} \rightarrow v_{*}^{\prime} \text { weakly in } L_{4 / 3}\left(0, T ; V^{-1}\right) \text { as } m \rightarrow \infty,
\end{gathered}
$$

and that the limit function $v_{*}$ belongs to the space $W_{1}$.

Consider Cauchy problem (3) for the limit function $v_{*}$. Since $v_{*} \in W_{1}$, therefore $v_{*}$ satisfies the conditions of Theorem 1 . Therefore, in $[0, T] \times[0, T] \times \bar{\Omega}$ there exists a Lagrangian regular flow $z_{*}(\tau ; t, x)$ associated to $v_{*}$. We denote by $z_{m}(\tau ; t, x)$ the Lagrangian regular flow associated to $v_{m}$.

Lemma 8. The sequence $z_{m}(\tau ; t, x)$ converges to $z(\tau ; t, x)$ with respect to the Lebesgue measure on the set $[0, T] \times \Omega$ in $(\tau, x)$ for $t \in[0, T]$.

This lemma follows from the a priori estimate (42) and Theorem 2.

The proofs of the solvability of feedback control problem (8), (1)-(5) are divided into two parts. First, we prove the passage to the limit in auxiliary problem (8), (10)-(14) for $\xi=1$ and a test function $\varphi$ from $V^{1}$, which is sufficiently smooth, then for the arbitrary function $\varphi \in V^{1}$.

I part. Let the test function $\varphi \in V^{1}$ be smooth. We pass to the limit in each term of (43).

For $m \rightarrow \infty$, by the definition of weak convergence $v_{m} \rightarrow v^{*}$ in $L_{2}\left(0, T ; V^{1}\right)$ we get

$$
\mu_{0} \int_{\Omega} \nabla v_{m}: \nabla \varphi d x \rightarrow \mu_{0} \int_{\Omega} \nabla v_{*}: \nabla \varphi d x
$$

for any $\varphi \in V^{1}$.

Due to weak convergence $v_{m}^{\prime} \rightarrow v_{*}^{\prime}$ in $L_{4 / 3}\left(0, T ; V^{-1}\right)$ as $m \rightarrow \infty$ we obtain that

$$
\left\langle v_{m}^{\prime}, \varphi\right\rangle \rightarrow\left\langle v_{*}^{\prime}, \varphi\right\rangle
$$

for any $\varphi \in V^{1}$.

Further, using estimate (47), without loss of generality and, if necessary, passing to a subsequence, we have that there exists a function $u \in L_{4 / 3}\left(0, T ; V^{3}\right)$ such that

$$
\varepsilon_{m} v_{m}^{\prime} \rightarrow u \text { weakly in } L_{4 / 3}\left(0, T ; V^{3}\right) \text { as } m \rightarrow \infty .
$$

Then

$$
\varepsilon_{m}\left\langle\nabla \Delta v_{m}^{\prime}, \nabla \varphi\right\rangle \rightarrow\langle\nabla \Delta u, \nabla \varphi\rangle, \quad \text { as } \quad m \rightarrow \infty .
$$

However, the sequence $\varepsilon_{m} v_{m}^{\prime}$ converges to zero in the sense of distributions on $[0, T]$ with values in $V^{-3}$. Indeed, for any smooth scalar function $\psi$ with compact support and for $\varphi \in V^{3}$, we obtain 


$$
\begin{gathered}
\lim _{m \rightarrow \infty}\left|\varepsilon_{m} \int_{0}^{T} \int_{\Omega} \nabla \Delta v_{m}^{\prime}: \nabla \varphi d x \psi(t) d t\right|=\lim _{m \rightarrow \infty} \varepsilon_{m}\left|\int_{0}^{T} \int_{\Omega} \Delta v_{m}^{\prime} \Delta \varphi d x \psi(t) d t\right| \\
=\lim _{m \rightarrow \infty} \varepsilon_{m}\left|\int_{0}^{T} \int_{\Omega} \nabla v_{m}^{\prime}: \nabla \Delta \varphi d x \psi(t) d t\right|=\lim _{m \rightarrow \infty} \varepsilon_{m} \lim _{m \rightarrow \infty}\left|\int_{0}^{T} \int_{\Omega} \nabla v_{m}^{\prime}: \nabla \Delta \varphi d x \psi(t) d t\right| \\
=\lim _{m \rightarrow \infty} \varepsilon_{m} \lim _{m \rightarrow \infty}\left|\int_{\Omega}\left(\int_{0}^{T} \nabla v_{m}^{\prime} \psi(t) d t\right): \nabla \Delta \varphi d x\right| \\
=\lim _{m \rightarrow \infty} \varepsilon_{m} \lim _{m \rightarrow \infty}\left|\int_{\Omega}\left(\int_{0}^{T} \nabla v_{m} \frac{\partial \psi(t)}{\partial t} d t\right): \nabla \Delta \varphi d x\right| \\
=\lim _{m \rightarrow \infty} \varepsilon_{m} \lim _{m \rightarrow \infty}\left|\int_{0}^{T} \int_{\Omega} \nabla v_{m}: \nabla \Delta \varphi d x \frac{\partial \psi(t)}{\partial t} d t\right| .
\end{gathered}
$$

Since $v_{m}$ weakly converges to $v^{*}$ in $L_{2}\left(0, T ; V^{1}\right)$ and, therefore, converges to $v^{*}$ in the sense of distributions, then

$$
\lim _{m \rightarrow \infty} \varepsilon_{m} \lim _{m \rightarrow \infty}\left|\int_{0}^{T} \int_{\Omega} \nabla v_{m}: \nabla \Delta \varphi d x \psi(t) d t\right|=\left|\int_{0}^{T} \int_{\Omega} \nabla v_{*}: \nabla \Delta \varphi d x \frac{\partial \psi(t)}{\partial t} d t\right| \lim _{m \rightarrow \infty} \varepsilon_{m}=0 .
$$

Thus, due to the uniqueness of the weak limit

$$
\varepsilon_{m}\left\langle\nabla \Delta v_{m}^{\prime}, \nabla \varphi\right\rangle \rightarrow 0 \quad \text { as } \quad m \rightarrow \infty .
$$

Since the embedding $V^{1} \subset L_{4}(\Omega)$ is completely continuous, and the embedding $L_{4}(\Omega) \subset$ $V^{-1}$ is continuous, by Theorem 5 it follows, that

$$
F=\left\{v \in L_{2}\left(0, T ; V^{1}\right), v^{\prime} \in L_{4 / 3}\left(0, T ; V^{-1}\right)\right\} \subset L_{2}\left(0, T ; L_{4}(\Omega)\right) .
$$

Then, taking into account estimates (45) and (46) we conclude that

$$
v_{m} \rightarrow v_{*} \text { strongly in } L_{2}\left(0, T ; L_{4}(\Omega)\right) .
$$

Since the operator $\Delta_{\alpha}^{-1}=\left(I-\alpha^{2} \Delta\right)^{-1}: L_{2}\left(0, T ; V^{1}\right) \rightarrow L_{2}\left(0, T ; V^{3}\right)$ is continuous, then

$$
\int_{\Omega} \sum_{i, j=1}^{n}\left(\Delta_{\alpha}^{-1} v_{m}\right)_{i}\left(v_{m}\right)_{j} \frac{\partial \varphi_{j}}{\partial x_{i}} d x \rightarrow \int_{\Omega} \sum_{i, j=1}^{n}\left(\Delta_{\alpha}^{-1} v_{*}\right)_{i}\left(v_{*}\right)_{j} \frac{\partial \varphi_{j}}{\partial x_{i}} d x \quad \text { as } \quad m \rightarrow \infty,
$$

where the first sequence $\left(\Delta_{\alpha}^{-1} v_{m}\right)_{i}$ weakly converges in $L_{2}\left(0, T ; V^{1}\right)$, and the second $\left(v_{m}\right)_{j}$ strongly in $L_{2}\left(0, T ; L_{4}(\Omega)\right)$. Consequently, their product converges weakly to the product of limits.

Now show that

$$
\begin{aligned}
\frac{\mu_{1}}{\Gamma(1-\beta)}\left(\int_{0}^{t}(t-s)^{-\beta} \mathcal{E}\left(v_{m}\right)\right. & \left.\left(s, z_{m}(s ; t, x)\right) d s, \mathcal{E}(\varphi)\right) \\
\rightarrow & \frac{\mu_{1}}{\Gamma(1-\beta)}\left(\int_{0}^{t}(t-s)^{-\beta} \mathcal{E}\left(v_{*}\right)\left(s, z_{*}(s ; t, x)\right) d s, \mathcal{E}(\varphi)\right) .
\end{aligned}
$$


Consider the following difference

$$
\begin{gathered}
\frac{\mu_{1}}{\Gamma(1-\beta)}\left(\int_{0}^{t}(t-s)^{-\beta} \mathcal{E}\left(v_{m}\right)\left(s, z_{m}(s ; t, x)\right) d s, \mathcal{E}(\varphi)\right) \\
-\frac{\mu_{1}}{\Gamma(1-\beta)}\left(\int_{0}^{t}(t-s)^{-\beta} \mathcal{E}\left(v_{*}\right)\left(s, z_{*}(s ; t, x)\right) d s, \mathcal{E}(\varphi)\right) \\
=\frac{\mu_{1}}{\Gamma(1-\beta)}\left(\int_{0}^{t}(t-s)^{-\beta} \int_{\Omega}\left[\mathcal{E}\left(v_{m}\right)\left(s, z_{m}(s ; t, x)\right)-\mathcal{E}\left(v_{*}\right)\left(s, z_{m}(s ; t, x)\right)\right]: \mathcal{E}(\varphi) d x d s\right) \\
+\frac{\mu_{1}}{\Gamma(1-\beta)}\left(\int_{0}^{t}(t-s)^{-\beta} \int_{\Omega}\left[\mathcal{E}\left(v_{*}\right)\left(s, z_{m}(s ; t, x)\right)-\mathcal{E}\left(v_{*}\right)\left(s, z_{*}(s ; t, x)\right)\right]: \mathcal{E}(\varphi) d x d s\right)=Z_{1}^{m}+Z_{2}^{m} .
\end{gathered}
$$

(1) We show first that $Z_{1}^{m} \rightarrow 0$ as $m \rightarrow \infty$.

Denote the integral over domain $\Omega$ in $Z_{1}^{m}$ by $I$ :

$$
I=\int_{\Omega}\left[\mathcal{E}\left(v_{m}\right)\left(s, z_{m}(s ; t, x)\right)-\mathcal{E}\left(v_{*}\right)\left(s, z_{m}(s ; t, x)\right)\right]: \mathcal{E}(\varphi) d x .
$$

We make the change of variables $x=z^{m}(t ; s, y)$ in $I$ (where the reverse change is $y=$ $\left.z_{m}(s ; t, x)\right)$ :

$$
I=\int_{\Omega}\left[\mathcal{E}\left(v_{m}\right)(s, y)-\mathcal{E}\left(v_{*}\right)(s, y)\right]: \mathcal{E}(\varphi)\left(z_{m}(t ; s, y)\right) d y .
$$

We rewrite $Z_{1}^{m}$ and continue the further expansion:

$$
\begin{gathered}
Z_{1}^{m}=\frac{\mu_{1}}{\Gamma(1-\beta)}\left(\int_{0}^{t}(t-s)^{-\beta} \int_{\Omega}\left[\mathcal{E}\left(v_{m}\right)(s, y)-\mathcal{E}\left(v_{*}\right)(s, y)\right]: \mathcal{E}(\varphi)\left(z_{m}(t ; s, y)\right) d y d s\right) \\
=\frac{\mu_{1}}{\Gamma(1-\beta)}\left(\int_{0}^{t}(t-s)^{-\beta} \int_{\Omega}\left[\mathcal{E}\left(v_{m}\right)(s, y)-\mathcal{E}\left(v_{*}\right)(s, y)\right]:\left[\mathcal{E}(\varphi)\left(z_{m}(t ; s, y)\right)\right.\right. \\
\left.\left.-\mathcal{E}(\varphi)\left(z_{*}(t ; s, y)\right)\right] d y d s\right)+\frac{\mu_{1}}{\Gamma(1-\beta)}\left(\int _ { 0 } ^ { t } ( t - s ) ^ { - \beta } \int _ { \Omega } \left[\mathcal{E}\left(v_{m}\right)(s, y)\right.\right. \\
\left.\left.-\mathcal{E}\left(v_{*}\right)(s, y)\right]: \mathcal{E}(\varphi)\left(z_{*}(t ; s, y)\right) d y d s\right)=Z_{11}^{m}+Z_{12}^{m}
\end{gathered}
$$

(a) Due to the weak convergence $v_{m}$ to $v_{*}$ in the space $L_{2}\left(0, T ; V^{1}\right)$, we obtain that $Z_{12}^{m} \rightarrow 0$ as $m \rightarrow \infty$.

(b) Applying the Holder and the Cauchy-Bunyakovsky inequalities, we get

$$
\begin{gathered}
\left|Z_{11}^{m}\right|^{2} \leq C_{31}\left(\int_{0}^{t}(t-s)^{-\beta}\left\|v_{m}(s, \cdot)-v_{*}(s, \cdot)\right\|_{V^{1}}\left\|\varphi_{x}\left(z_{m}(t ; s, \cdot)\right)-\varphi_{x}\left(z_{*}(t ; s, \cdot)\right)\right\|_{V^{0}} d s\right)^{2} \\
\leq C_{32}\left\|v_{m}(s, \cdot)-v_{*}(s, \cdot)\right\|_{L_{2}\left(0, T ; V^{1}\right)} \times \int_{0}^{T}\left\|\varphi_{x}\left(z_{m}(t ; s, \cdot)\right)-\varphi_{x}\left(z_{*}(t ; s, \cdot)\right)\right\|_{V^{0}} d s .
\end{gathered}
$$

We denote the second efficient in the last inequality by $\Phi_{m}(s)$ :

$$
\Phi_{m}(s)=\int_{0}^{T}\left\|\varphi_{x}\left(z_{m}(t ; s, \cdot)\right)-\varphi_{x}\left(z_{*}(t ; s, \cdot)\right)\right\|_{V^{0}} d s .
$$

We show the convergence $\Phi_{m}(s) \rightarrow 0$ as $m \rightarrow \infty$ for every $s \in[0, T]$. Note, that

$$
\Phi_{m}(s)=\int_{0}^{T} \int_{\Omega}\left|\varphi_{x}\left(z_{m}(t ; s, y)\right)-\varphi_{x}\left(z_{*}(t ; s, y)\right)\right|^{2} d y d s .
$$


Let $\varepsilon>0$ be a sufficiently small number.The continuity of the function $\varphi_{x}$ in $\bar{\Omega}$ means that there exists $\delta(\varepsilon)$ such that if $\left|x^{\prime \prime}-x^{\prime}\right| \leq \delta(\varepsilon)$, then

$$
\left|\varphi_{x}\left(x^{\prime \prime}\right)-\varphi_{x}\left(x^{\prime}\right)\right| \leq \varepsilon .
$$

Since the sequence $z_{m}(t ; s, y)$ converges to $z_{*}(t ; s, y)$ in the Lebesgue measure with respect to $(t, y)$, therefore for $\delta(\varepsilon)$ there exists the number $N=N(\delta(\varepsilon))$ which for $m \geq N$ the following inequality holds:

$$
m\left(\left\{(t, y):\left|z_{m}(t ; s, y)-z_{*}(t ; s, y)\right| \geq \delta(\varepsilon)\right\}\right) \leq \varepsilon .
$$

We denote

$$
\begin{aligned}
& Q(>\delta(\varepsilon))=\left\{(t, y) \in Q_{T}:\left|z_{m}(t ; s, y)-z_{*}(t ; s, y)\right|>\delta(\varepsilon)\right\} \\
& Q(\leq \delta(\varepsilon))=\left\{(t, y) \in Q_{T}:\left|z_{m}(t ; s, y)-z_{*}(t ; s, y)\right| \leq \delta(\varepsilon)\right\} .
\end{aligned}
$$

Then

$$
\begin{aligned}
\Phi_{m}(s) \leq & C_{33}\left(\int_{Q(>\delta(\varepsilon))}\left|\varphi_{x}\left(z_{m}(t ; s, y)\right)-\varphi_{x}\left(z_{*}(t ; s, y)\right)\right|^{2} d y d s\right. \\
& \left.\cdot \int_{Q(\leq \delta(\varepsilon))}\left|\varphi_{x}\left(z_{m}(t ; s, y)\right)-\varphi_{x}\left(z_{*}(t ; s, y)\right)\right|^{2} d y d s\right)=C_{33}\left(\Phi_{m}^{1}(s)+\Phi_{m}^{2}(s)\right) .
\end{aligned}
$$

By virtue of (53) for $\Phi_{m}^{2}(s)$ we have $\left|z_{m}(t ; s, y)-z_{*}(t ; s, y)\right| \leq \delta(\varepsilon)$. Hence

$$
\Phi_{m}^{2}(s) \leq \int_{Q(\leq \delta(\varepsilon))} \varepsilon^{2} d y d s=C_{34} \varepsilon^{2} .
$$

By virtue of (54) for $\Phi_{m}^{1}(s)$ we have $m(Q(>\delta(\varepsilon))) \leq \varepsilon$. Hence

$$
\Phi_{m}^{1}(s) \leq C_{35}\left\|\varphi_{x}\right\|_{C(\Omega)} \int_{Q(>\delta(\varepsilon))} d y d s=C_{35} \varepsilon\left\|\varphi_{x}\right\|_{C(\Omega)} .
$$

Thus, from (55), (56) and (57) it follows that for small $\varepsilon>0$ and $m \geq N(\delta(\varepsilon))$ the following inequality holds

$$
\Phi_{m}(s) \leq C_{36} \varepsilon
$$

Consequently, convergence $\Phi_{m}(s) \rightarrow 0$ as $m \rightarrow \infty$ for all $s \in[0, T]$ is obtained. Consider the right side of inequality (4). Due to the boundedness of the first efficient (since $v_{m} \in$ $\left.L_{2}\left(0, T ; V^{1}\right)\right)$ and the convergence to 0 of the second efficient as $m \rightarrow \infty$, we get that $Z_{11}^{m} \rightarrow 0$ as $m \rightarrow \infty$.

Thus, it is proved that $Z_{1}^{m} \rightarrow 0$ as $m \rightarrow \infty$.

(2) Now show that $Z_{2}^{m} \rightarrow 0$ as $m \rightarrow \infty$. Consider the auxiliary function $\widetilde{v}(t, x)$ smooth and finite on $[0, T] \times \Omega$ such that $\left\|v_{*}-\widetilde{v}\right\|_{L_{2}\left(0, T ; V^{1}\right)} \leq \varepsilon$ for sufficiently small $\varepsilon>0$. We now estimate $Z_{2}^{m}$ through three integrals

$$
\begin{aligned}
&\left|Z_{2}^{m}\right| \leq C_{37}\left(\int_{0}^{t}(t-s)^{-\beta} \int_{\Omega}\left\|v_{*}\left(s, z_{m}(s ; t, x)\right)-\widetilde{v}\left(s, z_{m}(s ; t, x)\right)\right\|_{V^{1}} d s\right. \\
&+\int_{0}^{t}(t-s)^{-\beta} \int_{\Omega}\left\|\widetilde{v}\left(s, z_{m}(s ; t, x)\right)-\widetilde{v}\left(s, z_{*}(s ; t, x)\right)\right\|_{V^{1}} d s \\
&\left.+\int_{0}^{t}(t-s)^{-\beta} \int_{\Omega}\left\|\widetilde{v}\left(s, z_{*}(s ; t, x)\right)-v_{*}\left(s, z_{*}(s ; t, x)\right)\right\|_{V^{1}} d s\right)=C_{37}\left(Z_{21}^{m}+Z_{22}^{m}+Z_{23}^{m}\right) .
\end{aligned}
$$


We make a change of variables in the norms under the integrals $Z_{21}^{m}$ and $Z_{23}^{m}$ :

$$
\begin{aligned}
\left\|v_{*}\left(s, z_{m}(s ; t, x)\right)-\widetilde{v}\left(s, z_{m}(s ; t, x)\right)\right\|_{V^{1}} & =\left\|v_{*}(s, y)-\widetilde{v}(s, y)\right\|_{V^{1}} \\
\left\|\widetilde{v}\left(s, z_{*}(s ; t, x)\right)-v_{*}\left(s, z_{*}(s ; t, x)\right)\right\|_{V^{1}} & =\left\|\widetilde{v}(s, y)-v_{*}(s, y)\right\|_{V^{1}}
\end{aligned}
$$

Then we get

$$
Z_{21}^{m}+Z_{23}^{m}=C_{37}\left(\int_{0}^{t}(t-s)^{-\beta}\left\|v_{*}(s, \cdot)-\widetilde{v}(s, \cdot)\right\|_{V^{1}} d s\right) \leq C_{37} \mathcal{\varepsilon}
$$

We estimate also $Z_{22}^{m}$

$$
Z_{22}^{m} \leq C_{37}\left(\int_{0}^{t}(t-s)^{-\beta}\left(\int_{\Omega}\left|\widetilde{v}_{x}\left(s, z_{m}(s ; t, \cdot)\right)-\widetilde{v}_{x}\left(s, z_{*}(s ; t, \cdot)\right)\right|^{2} d x\right)^{1 / 2} d s\right) .
$$

By virtue of Lemma $8 z_{m}(s ; t, x)$ converges to $z(s ; t, x)$ and the function $\widetilde{v}_{x}(t, x)$ is bounded and smooth. Therefore, by the Lebesgue theorem, we obtain that $Z_{2}^{m} \rightarrow 0$ as $m \rightarrow \infty$. Thus, convergence (51) is proved.

Taking into account the a priori estimates (44)-(46) and conditions ( $\Psi 1)-(\Psi 4)$, without loss of generality, we can assume that there exists $f_{*} \in L_{2}\left(0, T ; V^{-1}\right)$ such that $f_{m} \rightarrow f_{*} \in \Psi\left(v_{*}\right)$ as $m \rightarrow \infty$.

As a result, it was shown that the functions $v_{*}$ and $f_{*}$ with a smooth test function $\varphi$ from $V^{1}$ satisfy the equality:

$$
\begin{aligned}
\left\langle v_{*}^{\prime}, \varphi\right\rangle-\int_{\Omega} \sum_{i, j=1}^{n}\left(\Delta_{\alpha}^{-1} v_{*}\right)_{i}\left(v_{*}\right)_{j} \frac{\partial \varphi_{j}}{\partial x_{i}} d x+\mu_{0} \int_{\Omega} \nabla v_{*}: \nabla \varphi d x \\
\quad+\frac{\mu_{1}}{\Gamma(1-\beta)}\left(\int_{0}^{t}(t-s)^{-\beta} \mathcal{E}\left(v_{*}\right)\left(s, z_{*}(s ; t, x)\right) d s, \mathcal{E}(\varphi)\right)=\left\langle f_{*}, \varphi\right\rangle .
\end{aligned}
$$

Since the sequence $\left\{v_{m}\right\}$ has a priori estimates (44), (45) and (46), due to the weak convergence properties for $v_{*}$ we immediately obtain the estimate:

$$
\left\|v_{*}\right\|_{L_{\infty}\left(0, T ; V^{0}\right)}+\left\|v_{*}\right\|_{L_{2}\left(0, T ; V^{1}\right)}+\left\|v_{*}\right\|_{L_{4 / 3}\left(0, T ; V^{-1}\right)} \leq C_{38} .
$$

Whence it follows that $v_{*} \in W_{1}$. Thus, the passage to the limit was proved for a test function $\varphi \in V^{1}$, which is smooth.

II part. Let us prove this passage to the limit for an arbitrary test function $\varphi$ from $V^{1}$. We rewrite (58) for smooth $\varphi$ in the form:

$$
\left[G_{1}, \varphi\right]-\left[G_{2}, \varphi\right]=0
$$

where

$$
\begin{gathered}
{\left[G_{1}, \varphi\right]=\left\langle v^{\prime}, \varphi\right\rangle-\int_{\Omega} \sum_{i, j=1}^{n}\left(\Delta_{\alpha}^{-1} v\right)_{i}(v)_{j} \frac{\partial \varphi_{j}}{\partial x_{i}} d x+\mu_{0} \int_{\Omega} \nabla v: \nabla \varphi d x} \\
+\frac{\mu_{1}}{\Gamma(1-\beta)}\left(\int_{0}^{t}(t-s)^{-\beta} \mathcal{E}(v)(s, z(s ; t, x)) d s, \mathcal{E}(\varphi)\right) ;\left[G_{2}, \varphi\right]=\langle f, \varphi\rangle .
\end{gathered}
$$

Lemma 9. Let the test function $\varphi$ be smooth. Then

$$
\left|\left[G_{1}, \varphi\right]\right| \leq C_{39}\|\varphi\|_{V^{1}}, \quad\left|\left[G_{2}, \varphi\right]\right| \leq C_{40}\|\varphi\|_{V^{1}} .
$$

The proof of this Lemma is similar to obtaining a priori estimates in section 3. 
Since the set of smooth functions is dense in $V^{1}$, for $\varphi \in V^{1}$ there exists a sequence of smooth functions $\varphi^{l} \in V^{1}$ such that $\left|\varphi^{l}-\varphi\right|_{V^{1}} \rightarrow 0$ for $l \rightarrow \infty$. By virtue of (59) we obtain

$$
\begin{aligned}
{\left[G_{1}, \varphi\right]-\left[G_{2}, \varphi\right]=} & {\left[G_{1}, \varphi-\varphi^{l}\right]-\left[G_{2}, \varphi-\varphi^{l}\right]+\left[G_{1}, \varphi^{l}\right]-\left[G_{2}, \varphi^{l}\right] } \\
& =\left[G_{1}, \varphi-\varphi^{l}\right]-\left[G_{2}, \varphi-\varphi^{l}\right] .
\end{aligned}
$$

From the last equality and estimates (60) we obtain

$$
\left|\left[G_{1}, \varphi\right]-\left[G_{2}, \varphi\right]\right| \leq C_{41}\left|\varphi-\varphi^{l}\right| .
$$

Taking into account the last inequality and passing to the limit as $l \rightarrow \infty$ in equality (58) for $\varphi=\varphi^{l}$ we obtain equality (58) for arbitrary $\varphi \in V^{1}$, which completes the proof of the existence of weak solutions for feedback control problem (1)-(5), (8).

\section{Proof of Theorem 4}

From Theorem 3 we obtain that the set of solutions is nonempty. Therefore, there exists a minimizing sequence $\left(v_{l}, f_{l}\right) \in \Sigma$ such that

$$
\lim _{l \rightarrow \infty} \Phi\left(v_{l}, f_{l}\right)=\inf _{(v, f) \in \Sigma} \Phi(v, f) .
$$

As before, in the proof of Theorem 3 from estimates (44)-(46) it follows:

$$
\begin{gathered}
v_{l} \rightarrow v_{*} \text { weakly in } L_{2}\left(0, T ; V^{1}\right), \\
v_{l} \rightarrow{ }^{*} \text {-weakly in } L_{\infty}\left(0, T ; V^{0}\right), \\
v_{l}^{\prime} \rightarrow v_{*}^{\prime} \text { weakly in } L_{4 / 3}\left(0, T ; V^{-1}\right), \\
v_{l} \rightarrow v_{*} \text { strongly in } L_{2}\left(0, T ; L_{4}(\Omega)\right),
\end{gathered}
$$

$z_{l}(\tau ; t, x) \rightarrow z(\tau ; t, x)$ in the Lebesgue measure with respect to $(\tau, x)$ on $[0, T] \times \Omega$, $f_{l} \rightarrow f_{*} \in \Psi\left(v_{*}\right)$ strongly in $L_{2}\left(0, T ; V^{-1}\right)$.

Similarly from inclusion

$$
J v_{l}^{\prime}+\mu_{0} A v_{l}-B\left(v_{l}\right)+\frac{\mu_{1}}{\Gamma(1-\beta)} C\left(v_{l}, z_{l}\right)=f_{l} \in \Psi\left(v_{l}\right),
$$

passing to the limit, we obtain

$$
J v_{*}^{\prime}+\mu_{0} A v_{*}-B\left(v_{*}\right)+\frac{\mu_{1}}{\Gamma(1-\beta)} C\left(v_{*}, z_{*}\right)=f_{*} \in \Psi\left(v_{*}\right) .
$$

We get that $\left(v_{*}, f_{*}\right) \in \Sigma$. Since the functional $\Phi$ is lower semicontinuous with respect to the relatively weak topology, we have

$$
\Phi\left(v_{*}, f_{*}\right) \leq \inf _{(v, f) \in \Sigma} \Phi(v, f) .
$$

Thereby $\left(v_{*}, f_{*}\right)$ is the required solution. The theorem is proved.

\section{Conclusions}

To summarize all reasonings, calculations and proofs in this paper, the mathematical model describing the motion of viscoelastic mediums was investigated. This model is equipped with the Voigt rheological relation. This relation is considered with the left-side fractional Riemann-Liouville 
derivative, which allows us to take into account the memory of the medium. This memory is considered along the trajectory of the motion of fluid particles, determined by the velocity field. This allows a more accurate description of the physical process of fluid motion. Also in this paper the model under consideration is called the alpha-model. Interest in the study of alpha-models is primarily associated with their application to the study of turbulence effects for fluid flows.

The main result of this paper is the solutions existence to the feedback control problem for the mathematical model under consideration. Also the existence of an optimal solution to the problem under consideration that gives a minimum to a given bounded quality functional is proved. Results of this paper provide an opportunity for the future investigation of this model. The authors propose the following future research directions for the model under consideration-1) the numerical analysis of the obtained solutions; 2) the consideration of a turbulence case of this problem; 3) the investigation of a II class of alpha-models for this problem and so forth.

Author Contributions: Conceptualization, V.Z.; methodology, V.Z.; supervision, V.Z.; investigation, V.Z., A.Z. and A.U.; writing - original draft, review and editing, V.Z., A.Z. and A.U. All authors have read and agreed to the published version of the manuscript.

Funding: The work of the first author was supported by the Russian Foundation for Basic Research (project no. 20-01-00051, Lemma 4) and by the Ministry of Science and Higher Education of the Russian Federation (project no. FZGU-2020-0035, Theorem 3). The work of the second author was supported by the Russian Foundation for Basic Research (project no. 19-31-60014, Theorem 4). The work of the third author was supported by the Russian Science Foundation (project no. 19-11-00146, Theorem 6).

Conflicts of Interest: The authors declare no conflict of interest.

\section{References}

1. Martinez-Garcia, M.; Zhang, Y.; Gordon, T. Memory Pattern Identification for Feedback Tracking Control in Human-Machine Systems. Hum. Factors 2019. doi:10.1177/0018720819881008. [CrossRef]

2. Zvyagin, V.; Obukhovskii, V.; Zvyagin, A. On inclusions with multivalued operators and their applications to some optimization problems. J. Fixed Point Theory Appl. 2014, 16, 27-82. doi:10.1007/S11784-015-0219-2. [CrossRef]

3. Zvyagin, A.V. Optimal Feedback Control for Leray and Navier-Stokes Alpha Models. Dokl. Math. 2019, 99, 299-302. doi:10.1134/S1064562419030190. [CrossRef]

4. Zvyagin, V.G.; Zvyagin, A.V.; Turbin, M.V. Optimal Feedback Control Problem for the Bingham Model with Periodical Boundary Conditions on Spatial Variables. J. Math. Sci. 2020, pp. 959-980. doi:10.1007/s10958-020-04667-7. [CrossRef]

5. Zvyagin, A.V. Optimal Feedback Control in the Stationary Mathematical Model of Low Concentrated Aqueous Polymer Solutions. Appl. Anal. 2013, 92, 1157-1168. doi:10.1080/00036811.2011.653795. [CrossRef]

6. Kilbas, A.A.; Srivastava, H.M.; Trujillo, J.J. Theory and Applications of Fractional Differential Equations; Elsevier: Amsterdam, The Netherlands, 2006; Volume 204.

7. Leray, J. Sur le mouvement d'un liquide visqueux emplissant l'espace. Acta Math. 1934, 63, $193-248$. doi:10.1007/BF02547354. [CrossRef]

8. Holm, D.D.; Marsden, J.E.; Ratiu, T.S. The Euler-Poincare models of ideal fluids with nonlinear dispersion. Phys. Rev. Lett. 1998, 80, 4173-4176. doi:10.1103/PhysRevLett.80.4173. [CrossRef]

9. Holm, D.D.; Marsden, J.E.; Ratiu, T.S. The Euler-Poincaré Equations and Semidirect Products with Applications to Continuum Theories. Adv. Math. 1998, 137, 1-81. doi:10.1006/aima.1998.1721. [CrossRef]

10. Chen, S.; Foias, C.; Holm, D.D.; Olson, E.; Titi, E.S.; Wynne, S. Camassa-Holm equations as a closure model for turbulent channel and pipe flow. Phys. Rev. Lett. 1998, 81, 5338-5341. doi:10.1103/PhysRevLett.81.5338. [CrossRef]

11. Lemarie-Rieusset, P.G. The Navier-Stokes Problem in the 21st Century; Taylor and Francis Group, CRC Press: Boca Raton, FL, USA, 2016.

12. Cheskidov, A.; Holm, D.D.; Olson, E.; Titi, E.S. On a Leray- $\alpha$ model of turbulence. Proc. R. Soc. A 2005, 461, 629-649. doi:10.1098/rspa.2004.1373. [CrossRef]

13. Zvyagin, A.V. Solvability of Thermoviscoelastic Problem for Leray Alpha-Model. Russ. Math. 2016, 60, 629-649. doi:10.3103/S1066369X16100091. [CrossRef] 
14. Foias, C.; Holm, D.D.; Titi, E.S. The three dimensional viscous Camassa-Holm equations, and their relation to the Navier-Stokes equations and turbulence theory. J. Dyn. Differ. Equ. 2002, 14, 1-35. doi:10.1023/A:1012984210582. [CrossRef]

15. Zvyagin, A.V.; Polyakov, D.M. On the solvability of the Jeffreys-Oldroyd- $\alpha$ model. Differ. Equ. 2016, 52, 761-766. doi:10.1134/S0012266116060069. [CrossRef]

16. Zvyagin, A.V.; Zvyagin, V.G.; Polyakov, D.M. On solvability of a fluid flow alpha-model with memory. Russ. Math. 2018, 6, 69-74. doi:10.3103/S1066369X18060075. [CrossRef]

17. Zvyagin, A.V.; Zvyagin, V.G.; Polyakov, D.M. Dissipative Solvability of an Alpha Model of Fluid Flow with Memory. Comput. Math. Math. Phys. 2019, 59, 1185-1198. doi:10.1134/s0965542519070133. [CrossRef]

18. Zvyagin, A.V. Weak solvability and convergence of solutions for the fractional Voigt-model of a viscoelastic medium. Russ. Math. Surv. 2019, 74, 549-551. doi:10.1070/RM9880. [CrossRef]

19. Zvyagin, V.; Orlov, V. Weak solvability of fractional Voigt model of viscoelasticity. Discrete Contin. Dyn. Syst. Ser. A 2018, 38, 6327-6350. doi:10.3934/dcds.2018270. [CrossRef]

20. Mainardi, F.; Spada, G. Creep, relaxation and viscosity properties for basic fractional models in rheology. Eur. Phys. J. Spec. Top. 2011, 193, 133-160. doi:10.1140/epjst/e2011-01387-1. [CrossRef]

21. Caputo, M.; Mainardi, F. A new dissipation model based on memory mechanism. Pure Appl. Geophys. 1971, 91, 134-147. doi:10.1007/BF00879562. [CrossRef]

22. Gladkov, S.O. Theory of One-Dimensional and Quasi-one-dimensional Heat Conduction. Tech. Phys. 1997, 42, 724-727. doi:10.1134/1.1258707. [CrossRef]

23. Gladkov, S.O. On the Theory of Hydrodynamic Phenomena in Quasi-one-dimensional Systems. Tech. Phys. 2001, 46, 1475-1477. doi:10.1134/1.1418518. [CrossRef]

24. Gladkov, S.O.; Bogdanova, S.B. The Heat-transfer Theory for Quasi-n-dimensional System. Physica B (Amst. Neth.) 2010, 405, 1973-1975. doi:10.1016/j.physb.2010.01.077. [CrossRef]

25. Gladkov, S.O.; Bogdanova, S.B. On Fractional Differentiation. Vestn. Samara Univ. Nat. Sci. Ser. 2018, 24, 7-13. doi:10.18287/2541-7525-2018-24-3-7-13. [CrossRef]

26. Gladkov, S.O.; Bogdanova, S.B. To the Question of Fractional Differentiation. Part II. Vestn. Samara Univ. Nat. Sci. Ser. 2019, 25, 7-11. doi:10.18287/2541-7525-2019-25-3-7-11. [CrossRef]

27. Fursikov, A.V. Optimal Control of Distributed Systems. Theory and Applications; American Mathematical Society: Providence, RI, USA, 2000; Volume 187.

28. Zvyagin, V.G.; Turbin, M.V. Mathematical Problems in Viscoelastic Hydrodynamics; Krasand URSS Russia: Moscow, Russia, 2012. (In Russian)

29. Orlov, V.P.; Sobolevskii, P.E. On mathematical models of a viscoelasticity with a memory. Differ. Integral Equ. 1991, 4, 103-115.

30. Zvyagin, V.G.; Dmitrienko, V.T. On weak solutions of a regularized model of a viscoelastic fluid. Differ. Equ. 2002, 38, 1731-1744. doi:10.1023/A:1023860129831. [CrossRef]

31. DiPerna, R.J.; Lions, P.L. Ordinary differential equations, transport theory and Sobolev spaces. Invent. Math. 1989, 98, 511-547. doi:10.1007/BF01393835. [CrossRef]

32. Crippa, G. The ordinary differential equation with non-Lipschitz vector fields. Boll. Unione Mat. Ital. 2008, 9, 333-348.

33. Crippa, G.; De Lellis, C. Estimates and regularity results for the DiPerna-Lions flow. J. Reine Angezw. Math. 2008, 2008, 15-46. doi:10.1515/CRELLE.2008.016. [CrossRef]

34. Temam, R. Navier-Stokes Equations: Theory and Numerical Analysis; AMS Chelsea: Providence, RI, USA, 2001.

35. Zvyagin, V.G. Topological approximation approach to study of mathematical problems of hydrodynamics. J. Math. Sci. 2014, 201, 830-858. doi:10.1007/s10958-014-2028-3. [CrossRef]

36. Turbin, M.V.; Ustiuzhaninova, A.S. The existence theorem for a weak solution to initial-boundary value problem for system of equations describing the motion of weak aqueous polymer solutions. Russ. Math. 2019, 63, 54-69. doi:10.3103/s1066369x19080061. [CrossRef]

37. Agranovich, M.S.; Vishik, M.I. Elliptic problems with a parameter and parabolic problems of general type. Russ. Math. Surv. 1964, 19, 53-157. doi:10.1070/RM1964v019n03ABEH001149. [CrossRef]

38. Agmon, S. On the eigenfunctions and on the eigenvalues of general elliptic boundary value problems. Commun. Pure Appl. Math. 1962, 15, 119-147. doi:10.1007/978-3-642-10994-2_1. [CrossRef]

39. Aubin, J.P. Un théorème de compacité. Comptes Rendus L'AcadéMie Des Sci. 1963, 256, 5042-5044. 
40. Simon, J. Compact sets in the spacel $L^{p}(0, T ; B)$. Ann. di Mat. Pura ed Appl. 1986, 146, 65-96. doi:0.1007/BF01762360. [CrossRef]

41. Samko, S.G.; Kilbas, A.A.; Marichev, O.I. Integrals and Fractional Derivatives and Some of Their Applications; Science and Technology: Minsk, Belarus, 1987. (In Russian).

42. Ahmerov, P.P.; Kamenskii, M.I.; Potapov, A.S.; Rodkina, A.E.; Sadovskii, B.N. Non-Compactness and Compacting Operators; Nauka: Novosibirsk, Russia, 1986. (In Russian)

43. Borisovich, Y.G.; Gel'man, B.D.; Myshkis, A.D.; Obukhovskii, V.V. Introduction to the Theory of Multivalued Mappings and Differential Inclusions; URSS: Moscow, Russia, 2011. (In Russian)

(C) 2020 by the authors. Licensee MDPI, Basel, Switzerland. This article is an open access article distributed under the terms and conditions of the Creative Commons Attribution (CC BY) license (http://creativecommons.org/licenses/by/4.0/). 